Provided for non-commercial research and education use. Not for reproduction, distribution or commercial use.

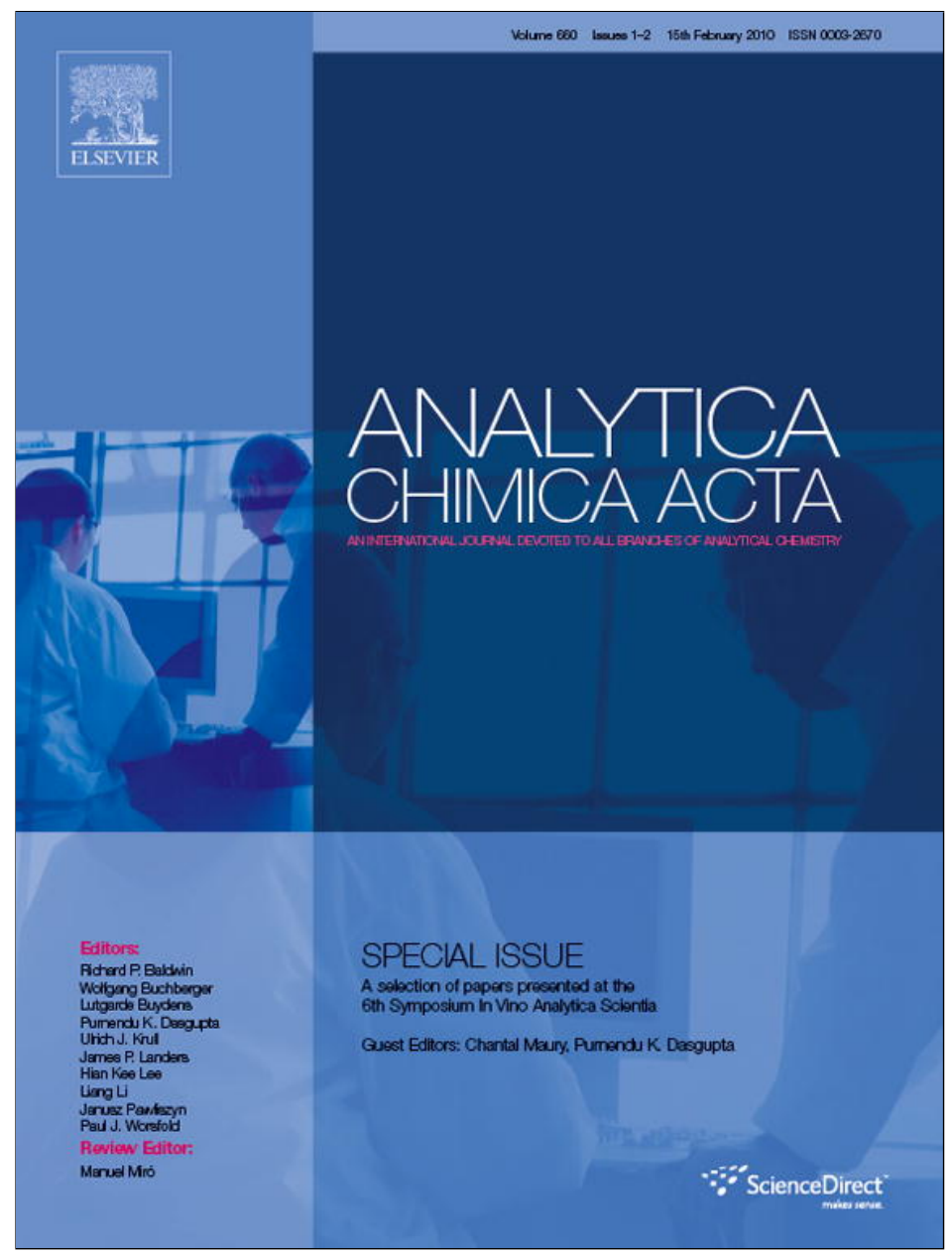

This article appeared in a journal published by Elsevier. The attached copy is furnished to the author for internal non-commercial research and education use, including for instruction at the authors institution and sharing with colleagues.

Other uses, including reproduction and distribution, or selling or licensing copies, or posting to personal, institutional or third party websites are prohibited.

In most cases authors are permitted to post their version of the article (e.g. in Word or Tex form) to their personal website or institutional repository. Authors requiring further information regarding Elsevier's archiving and manuscript policies are encouraged to visit:

http://www.elsevier.com/copyright 


\title{
Colour and pigment composition of red wines obtained from co-maceration of Tempranillo and Graciano varieties
}

\author{
Matilde García-Marino, José Miguel Hernández-Hierro, \\ Julián C. Rivas-Gonzalo, M. Teresa Escribano-Bailón* \\ Grupo de Investigación en Polifenoles, Unidad de Nutrición y Bromatología, Facultad de Farmacia, Universidad de Salamanca, Campus Miguel de Unamuno, \\ E 37007 Salamanca, Spain
}

\section{A R T I C L E I N F O}

\section{Article history:}

Received 28 July 2009

Received in revised form 6 October 2009

Accepted 22 October 2009

Available online 29 October 2009

\section{Keywords:}

Wine colour

Anthocyanins

Tempranillo

Graciano

Co-maceration

Principal component analysis

\begin{abstract}
A B S T R A C T
The Vitis vinifera L. cv Graciano is often used as an integral component of Rioja wines because it is considered to contribute significantly to the quality of Tempranillo based wines. The aim of this work was to determine the effect of the incorporation of the Graciano variety on the colour and pigment composition of Tempranillo based wine and to evaluate the possible differences between blending Tempranillo with Graciano varietal wine ( $\mathbf{W}$ wine) in contrast to a wine obtained by mixing these two grape varieties in the prefermentative maceration step (M wine). Results indicated that pigment extraction and retention in $\mathrm{M}$ wine was higher than in Tempranillo $(\mathbf{T})$ wine. Colour differences $\left(\Delta E_{\mathrm{ab}}^{*}\right)$ between wines at the end of the study (after 12 months in bottles) were detectable by the human eye $\left(\Delta E_{\mathrm{ab}}^{*} \geq 3\right)$ in all cases except for $\mathbf{T}$ and $\mathbf{W}$. These wines have indistinguishable colours even when using analytical methods $\left(\Delta E_{\mathrm{ab}}^{*} \leq 1\right)$. Regarding hue $h_{\mathrm{ab}}, \mathbf{T}$ and $\mathbf{W}$ presented higher values (more orange-red) than Graciano (G) and $\mathbf{M}$ (more blue-red). PCA allowed the colour and composition analytical data to be reduced to a small number of principal components that could separate successfully between $\mathbf{T}$ and $\mathbf{G}$ wines and between the different steps of the winemaking process.
\end{abstract}

(C) 2009 Elsevier B.V. All rights reserved.

\section{Introduction}

Red wine colour is strongly influenced by the phenolic content of the grapes, as well as oenological practices and storage conditions. It is usually accepted that the colour of young red wine is mainly due to the anthocyanin composition of grapes while the colour of aged red wine is a consequence of its instability and reactivity $[1,2]$. The copigmentation processes are also supposed to account for the colour of red wines [3,4] and they could also act as a first stage in the formation of new pigments that determine the colour of aged red wines [5]. It seems, therefore, that colour extraction and retention in the wine is greatly influenced by the levels of cofactors on it. For that reason some authors suggest the incorporation of extra copigments by external addition. The addition of caffeic and $p$-coumaric acids in the prefermentative step of the winemaking process enhances the intermolecular copigmentation observed in the final red wines [6-8]. Another practice that could contribute to increase the extent of the copigmentation process is the co-vinification of different grape varieties [9]. Lorenzo et al. [10] in studies where Monastrell grapes were co-fermented

\footnotetext{
* Corresponding author. Tel.: +34 923294 537; fax: +34 923294515

E-mail address: escriban@usal.es (M.T. Escribano-Bailón).
}

in the presence of Cabernet Sauvignon and Merlot grapes observed an increase in the phenolic extraction which influences the colour of the finished wine. Likewise, blends from different monovarietal wines give rise to wines with a more balanced anthocyanin/flavanol ratio [11].

The Vitis vinifera L. cv Tempranillo is the most widely grown red grape variety in Spain and it is cultivated in 61\% of the A.O.C. Rioja. V. vinifera L. cv Graciano is less known but is used as an integral component of many Rioja wines because it is considered to contribute significantly to the quality of the wine. This variety is related to Morrastel (France) and to Tinta Miúda (Portugal). Recent studies [12] showed that copigmentation and self-association processes take place more favourably in the essays carried out with Graciano variety than in those with Tempranillo.

Usually exploratory data analysis is a preliminary stage prior to supervised modelling such as classification (or calibration in other contexts) and can answer whether there are any groupings in the data, whether there are outliers, whether samples from a similar source are related, or even whether there are trends, such as temporal ones, in the data. Principal components analysis (PCA) is probably the most widespread multivariate chemometric technique. It is certainly the most widespread technique in multivariate exploratory data analysis $[13,14]$ and has been successfully applied to analytical results obtained from wines [15-17]. 
Table 1

Chromatographic and MS spectrum data of the anthocyanins detected as well as their identities.

\begin{tabular}{|c|c|c|c|c|}
\hline Pigments $(\lambda=520 \mathrm{~nm})$ & $\mathrm{Rt}_{\min }$ & Compounds & {$\left[\mathrm{M}^{+}\right](m / z)$} & $\mathrm{MS}^{2}$ (relative abundance) \\
\hline 1 & 10,019 & Direct condensation product between malvidin-3-O-glucoside and (epi)gallocatechin & 797 & $653(100)$ \\
\hline 2 & 11,459 & Delphinidin-3,5-diglucoside & 627 & 465(100), 303(73) \\
\hline 3 & 14,168 & Direct condensation product between petunidin-3-O-glucoside and (epi)catechin & 767 & $605(100), 285(40)$ \\
\hline 4 & 15,626 & Petunidin-3,5-diglucoside & 641 & $357(100), 479(20)$ \\
\hline 5 & 16,191 & Delphinidin-3,7-diglucoside & 627 & $465(100), 303(35)$ \\
\hline 6 & 16,783 & Vitisin A delphinidin-3-O-glucoside & 533 & $371(100), 525(7)$ \\
\hline 7 & 17,668 & Direct condensation product between peonidin-3-O-glucoside and (epi)catechin & 751 & $589(100), 571(6)$ \\
\hline 8 & 17,820 & Direct condensation product between malvidin-3-O-glucoside and (epi)catechin & 781 & 619(100), 373(13) \\
\hline 9 & 18,315 & Delphinidin-3-O-glucoside & 465 & $303(100)$ \\
\hline 10 & 20,725 & Petunidin-3,7-diglucoside & 641 & $317(100), 479(20)$ \\
\hline 11 & 22,134 & Cyanidin-3-O-glucoside & 449 & $287(100)$ \\
\hline 12 & 22,661 & Vitisin A petunidin-3-O-glucoside & 547 & $385(100)$ \\
\hline 13 & 23,653 & Petunidin-3-O-glucoside & 479 & $317(100)$ \\
\hline 14 & 25,927 & Malvidin-3,7-diglucoside & 655 & 331(100), 316(29), 493(19) \\
\hline 15 & 28,992 & Vitisin A peonidin-3-O-glucoside & 531 & $369(100), 502(6)$ \\
\hline 16 & 29,180 & Peonidin-3-O-glucoside & 463 & $301(100)$ \\
\hline 17 & 29,810 & Vitisin A malvidin-3-O-glucoside & 561 & $399(100), 510(2)$ \\
\hline 18 & 30,076 & Malvidin-3-O-glucoside & 493 & $331(100)$ \\
\hline 19 & 33,284 & Delphinidin-3-O-(6'-acetyl)-glucoside & 507 & $303(100)$ \\
\hline 20 & 33,740 & Malvidin-3-O-hexose & 493 & $331(100), 427(34)$ \\
\hline 21 & 34,839 & Vitisin B malvidin-3-O-glucoside & 517 & $355(100)$ \\
\hline 22 & 36,565 & Malvidin-3-O-pentose & 463 & 331(100), 332(4) \\
\hline 23 & 37,016 & Malvidin-3-O-glucoside-8-ethyl-(epi)catechin & 809 & $357(100), 519(63)$ \\
\hline 24 & 37,402 & Petunidin-3-O-(6'-acetyl)glucoside & 521 & $317(100)$ \\
\hline 25 & 37,885 & Malvidin-3-O-glucoside-8-ethyl-(epi)gallocatechin & 825 & 357(100), 663(52), 519(41) \\
\hline 26 & 38,391 & Malvidin-3-O-glucoside-8-ethyl-(epi)catechin & 809 & $357(100), 519(35)$ \\
\hline 27 & 38,660 & Cyanidin-3-O-hexose & 449 & $287(100)$ \\
\hline 28 & 39,309 & Malvidin-3-O-glucoside-8-ethyl-metoxiflavanol & 839 & $357(100), 677(37), 519(21)$ \\
\hline 29 & 39,949 & Malvidin-3-O-(6'-p-coumaroyl)glucoside-8-ethyl-(epi)catechin & 927 & $619(100), 373(75)$ \\
\hline 30 & 40,561 & Peonidin-3-O-(6'-acetyl)glucoside & 505 & $301(100)$ \\
\hline 31 & 40,760 & Vitisin A malvidin-3-O-(6'-p-coumaroyl)glucoside & 707 & $399(100), 383(2)$ \\
\hline 32 & 41,075 & Malvidin-3-O-(6'-acetyl)glucoside & 535 & $331(100)$ \\
\hline 33 & 41,227 & Delphinidin-3-O-(6'-p-coumaroyl)glucoside & 611 & $303(100), 257(2)$ \\
\hline 34 & 42,447 & Petunidin-3-O-(6'-p-coumaroyl)glucoside (cis) & 625 & $317(100), 492(10)$ \\
\hline 35 & 43,002 & Malvidin-3-O-(6'-p-caffeoyl)glucoside & 655 & $331(100), 316(50)$ \\
\hline 36 & 43,724 & Cyanidin-3-O-(6'-p-coumaroyl)glucoside & 595 & $287(100)$ \\
\hline 37 & 44,123 & Petunidin-3-O-(6'-p-coumaroyl)glucoside (trans) & 625 & $317(100), 302(7)$ \\
\hline 38 & 45,415 & Malvidin-3-O-(6'-p-coumaroyl)glucoside (cis) & 639 & $331(100), 315(5), 287(2)$ \\
\hline 39 & 46,179 & Peonidin-3-O-glucoside-4-vynilcatechol & 595 & $433(100), 408(11)$ \\
\hline 40 & 46,990 & Peonidin-3-O-(6'-p-coumaroyl)glucoside (trans) & 609 & 301(100), 286(53), 463(2) \\
\hline 41 & 47,040 & Malvidin-3-O-(6'-p-coumaroyl)glucoside (trans) & 639 & $331(100), 315(2)$ \\
\hline 42 & 49,517 & Malvidin-3-O-glucoside-4-vinilphenol & 609 & $447(100), 286(5)$ \\
\hline 43 & 50,357 & Malvidin-3-O-glucoside-4-vinilguaiacol & 639 & $477(100), 331(9)$ \\
\hline 44 & 52,833 & Malvidin-3-O-(6'-acetyl)glucoside-4-vinilphenol & 651 & $447(100)$ \\
\hline 45 & 55,161 & Malvidin-3-O-(6'-p-coumaroyl)glucoside-4-vinilphenol & 755 & $447(100), 431(4)$ \\
\hline 46 & 55,500 & Malvidin-3-O-(6'-p-coumaroyl)glucoside-4-vinilguaiacol & 785 & $477(100), 722(33)$ \\
\hline
\end{tabular}

$\mathrm{Rt}_{\min }$ : retention time in minutes; $\left[\mathrm{M}^{+}\right]$: positive charged molecular ion; $\mathrm{MS}^{2}$ : fragmentation of $\left[\mathrm{M}^{+}\right]$.

The aim of this study is to compare the pigment composition and colour of wines obtained by blending Tempranillo with Graciano varietal wines in contrast to wines obtained by mixing these two grape varieties in the prefermentative maceration step. Statistical correlations and principal components analysis (PCA) were applied in order to determine the relationship between pigment composition and colour and to identify the main factors/variables that explain most of the variance observed in the dataset.

\section{Materials and methods}

\subsection{Samples}

The wines used in this study were processed by Bodegas RODA (Haro, La Rioja, Spain). T and $\mathbf{G}$ wines were made from $V$. vinifera cv Tempranillo and Graciano fresh grapes, respectively. $\mathbf{M}$ wine results from a mixture of Tempranillo/Graciano (80:20) grapes and $\overline{\mathbf{W}}$ from a blending of $\mathbf{T}$ and $\mathbf{G}$ wines (80:20) after malolactic fermentation. Samples were collected periodically during winemaking and ageing and correspond to the following steps: after alcoholic fermentation (16 days, step 1 ), at the end of postfermentative maceration (20 days, step 2), at the beginning of malolactic fermentation (22 days, step 3), in mid malolactic fermentation (29 days, step 4), at the end of malolactic fermentation (39 days, step 5), after 3 months in oak barrels (oak barrels 128 days, step 6 ), after 6 months in oak barrels (219 days, step 7), after 10 months in oak barrels (413 days, step 8 ), after 12 months in oak barrel-bottling (460 days, step 9), after 5 months in bottles (602 days, step 10), after 9 months in bottles (736 days, step 11 ) and after 12 months in bottles (827 days, step 12). All samples were taken in triplicate and analyzed separately.

\subsection{HPLC-DAD analysis}

Hewlett-Packard 1100 equipment provided with a quaternary pump, automatic injector, DAD and data treatment station was used. An Aqua ${ }^{\circledR} \mathrm{C} 18$ column ( $4.6 \mathrm{~mm} \times 150 \mathrm{~mm}, 5 \mu \mathrm{m}$ particle size) thermostatted at $35^{\circ} \mathrm{C}$ was used. Solvents were (A) $0.1 \%$ trifluoroacetic acid, and (B) 100\% HPLC grade acetonitrile. The elution profile was as follows: isocratic $10 \% \mathrm{~B}$ for $3 \mathrm{~min}$, from 10 to $15 \% \mathrm{~B}$ for $12 \mathrm{~min}$, isocratic $15 \%$ B for $5 \mathrm{~min}$, from 15 to $18 \%$ B for $5 \mathrm{~min}$, from 18 to $30 \% \mathrm{~B}$ for $20 \mathrm{~min}$ and from 30 to $35 \% \mathrm{~B}$ for $5 \mathrm{~min}$. The flow-rate was $0.5 \mathrm{~mL} \mathrm{~min}^{-1}$ and the injection volume $100 \mu \mathrm{L}$. UV-vis spectra were recorded from 250 to $770 \mathrm{~nm}$ with a bandwidth of $2 \mathrm{~nm}$. The preferred detection wavelengths were 505 and $520 \mathrm{~nm}$. 
Table 2

Mean concentration ( $\mathrm{mg} \mathrm{L}^{-1}, \pm$ S.D.; $n=3$ ) of different pigment families during winemaking and aging of wines T, G, M and W.

\begin{tabular}{|c|c|c|c|c|c|c|c|c|c|c|c|c|c|c|c|c|c|c|c|c|c|c|c|c|}
\hline \multirow[t]{3}{*}{ Time (days) } & \multicolumn{24}{|c|}{ Pigments } \\
\hline & \multicolumn{3}{|c|}{$\begin{array}{l}\text { Anthocyanidin- } \\
\text { 3-O-glucosides }\end{array}$} & \multicolumn{3}{|c|}{$\begin{array}{l}\text { Anthocyanidin- } \\
\text { 3-O-diglucosides }\end{array}$} & \multicolumn{3}{|c|}{$\begin{array}{l}\text { Acylated } \\
\text { anthocyanins }\end{array}$} & \multicolumn{3}{|c|}{ Pyranoanthocyanins } & \multicolumn{3}{|c|}{$\begin{array}{l}\text { Condensation } \\
\text { derived pigments }\end{array}$} & \multicolumn{3}{|c|}{ Total athocyanins } & \multicolumn{3}{|c|}{$\begin{array}{l}\text { Total } \\
\text { anthocyanin } \\
\text { derivates }\end{array}$} & \multicolumn{3}{|c|}{ Total pigments } \\
\hline & Mean & \pm S.D. & & Mean & \pm S.D. & & Mean & \pm S.D. & & Mean & \pm S.D. & & Mean & \pm S.D. & & Mean & \pm S.D. & & Mean & \pm S.D. & & Mean & \pm S.D. & \\
\hline \multicolumn{25}{|c|}{ Wine: Tempranillo $(\mathrm{T})$} \\
\hline 16 & 1014.14 & 0.31 & $a / B$ & 36.03 & 0.15 & $\mathrm{ab} / \mathrm{AB}$ & 206.84 & 0.14 & $\mathrm{a} / \mathrm{C}$ & 61.32 & 2.12 & $\mathrm{a} / \mathrm{C}$ & 61.82 & 0.81 & $\mathrm{ab} / \mathrm{A}$ & 1257.01 & 0.60 & $a / C$ & 123.15 & 1.31 & $\mathrm{ab} / \mathrm{B}$ & 1380.16 & 1.91 & $\mathrm{a} / \mathrm{C}$ \\
\hline 20 & 937.95 & 8.27 & $\mathrm{~b} / \mathrm{c}$ & 38.13 & 0.31 & $\mathrm{a} / \mathrm{A}$ & 186.59 & 1.21 & $\mathrm{~b} / \mathrm{C}$ & 63.48 & 0.72 & $\mathrm{a} / \mathrm{B}$ & 57.64 & 0.18 & abcde/A & 1162.67 & 9.17 & $\mathrm{~b} / \mathrm{c}$ & 121.12 & 0.91 & $a b / B$ & 1283.79 & 10.07 & $\mathrm{~b} / \mathrm{C}$ \\
\hline 22 & 827.28 & 14.33 & $\mathrm{c} / \mathrm{C}$ & 36.03 & 0.35 & $a b / A$ & 166.02 & 3.68 & $\mathrm{c} / \mathrm{C}$ & 64.76 & 0.84 & $\mathrm{a} / \mathrm{B}$ & 55.61 & 1.43 & de/A & 1029.33 & 18.35 & $\mathrm{c} / \mathrm{C}$ & 120.37 & 0.59 & $\mathrm{ab} / \mathrm{B}$ & 1149.71 & 18.94 & $\mathrm{c} / \mathrm{C}$ \\
\hline 29 & 794.54 & 25.12 & $\mathrm{c} / \mathrm{C}$ & 35.87 & 0.27 & $a b / A$ & 163.23 & 0.86 & $\mathrm{c} / \mathrm{C}$ & 66.17 & 2.90 & $\mathrm{a} / \mathrm{A}$ & 56.85 & 1.81 & $\mathrm{cde} / \mathrm{B}$ & 993.63 & 26.26 & $\mathrm{c} / \mathrm{C}$ & 123.02 & 4.70 & $\mathrm{ab} / \mathrm{B}$ & 1116.65 & 30.96 & $\mathrm{c} / \mathrm{C}$ \\
\hline 39 & 682.55 & 58.08 & $\mathrm{~d} / \mathrm{B}$ & 37.21 & 0.86 & $\mathrm{a} / \mathrm{A}$ & 156.51 & 9.49 & $\mathrm{c} / \mathrm{C}$ & 65.93 & 4.08 & $\mathrm{a} / \mathrm{B}$ & 57.09 & 1.66 & bcde/A & 876.27 & 68.33 & $\mathrm{~d} / \mathrm{B}$ & 123.02 & 3.40 & $\mathrm{ab} / \mathrm{AB}$ & 999.29 & 69.38 & $\mathrm{~d} / \mathrm{B}$ \\
\hline 128 & 672.67 & 42.30 & $\mathrm{~d} / \mathrm{C}$ & 35.91 & 1.51 & $a b / A$ & 158.31 & 9.50 & $c / B$ & 64.22 & 2.13 & $\mathrm{a} / \mathrm{B}$ & 57.44 & 2.58 & bcde/A & 866.89 & 53.30 & $\mathrm{~d} / \mathrm{C}$ & 121.65 & 4.49 & $\mathrm{ab} / \mathrm{B}$ & 988.54 & 57.68 & $\mathrm{~d} / \mathrm{C}$ \\
\hline 219 & 559.01 & 40.29 & $\mathrm{e} / \mathrm{A}$ & 33.86 & 0.75 & $\mathrm{bc} / \mathrm{A}$ & 133.49 & 7.80 & $\mathrm{~d} / \mathrm{B}$ & 62.50 & 2.82 & $\mathrm{a} / \mathrm{A}$ & 53.02 & 1.47 & $\mathrm{e} / \mathrm{A}$ & 726.36 & 45.01 & $\mathrm{e} / \mathrm{A}$ & 115.52 & 4.17 & $\mathrm{~b} / \mathrm{A}$ & 841.87 & 49.13 & $\mathrm{e} / \mathrm{A}$ \\
\hline 413 & 452.27 & 1.44 & $\mathrm{f} / \mathrm{AB}$ & 35.64 & 1.21 & $\mathrm{ab} / \mathrm{A}$ & 121.04 & 4.26 & $\mathrm{de} / \mathrm{B}$ & 63.17 & 0.15 & $\mathrm{a} / \mathrm{A}$ & 61.80 & 3.29 & $\mathrm{ab} / \mathrm{A}$ & 608.95 & 6.91 & $\mathrm{f} / \mathrm{B}$ & 124.97 & 3.44 & $\mathrm{a} / \mathrm{A}$ & 733.92 & 10.35 & $f / B$ \\
\hline 460 & 435.14 & 5.40 & $\mathrm{f} / \mathrm{B}$ & 31.81 & 1.36 & $c / B$ & 134.14 & 9.50 & $\mathrm{~d} / \mathrm{A}$ & 66.43 & 1.84 & $\mathrm{a} / \mathrm{AB}$ & 61.21 & 0.58 & $\mathrm{abc} / \mathrm{A}$ & 601.09 & 13.40 & $\mathrm{f} / \mathrm{A}$ & 27.64 & 2.26 & $\mathrm{a} / \mathrm{AB}$ & 728.73 & 12.71 & $\mathrm{f} / \mathrm{A}$ \\
\hline 602 & 301.94 & 4.16 & $g / C$ & 28.90 & 1.09 & $\mathrm{~d} / \mathrm{A}$ & 117.82 & 4.29 & $\mathrm{de} / \mathrm{B}$ & 65.27 & 0.92 & $\mathrm{a} / \mathrm{A}$ & 61.53 & 0.68 & $\mathrm{abc} / \mathrm{A}$ & 448.66 & 8.62 & $\mathrm{~g} / \mathrm{B}$ & 126.81 & 0.64 & $\mathrm{a} / \mathrm{B}$ & 575.46 & 8.15 & $\mathrm{~g} / \mathrm{B}$ \\
\hline 736 & 206.08 & 7.69 & $\mathrm{~h} / \mathrm{C}$ & 28.69 & 0.46 & $\mathrm{~d} / \mathrm{A}$ & 113.84 & 5.77 & $\mathrm{e} / \mathrm{AB}$ & 66.13 & 0.46 & $\mathrm{a} / \mathrm{A}$ & 62.23 & 1.15 & $\mathrm{a} / \mathrm{AB}$ & 348.61 & 13.79 & $\mathrm{~h} / \mathrm{C}$ & 128.36 & 1.60 & $\mathrm{a} / \mathrm{AB}$ & 476.97 & 15.34 & $\mathrm{~h} / \mathrm{C}$ \\
\hline 827 & 171.50 & 0.54 & $\mathrm{~h} / \mathrm{D}$ & 27.82 & 0.40 & $d / B$ & 111.29 & 3.34 & $\mathrm{e} / \mathrm{A}$ & 65.53 & 1.42 & $\mathrm{a} / \mathrm{A}$ & 58.46 & 0.71 & abcd/A & 310.61 & 3.09 & $\mathrm{~h} / \mathrm{C}$ & 123.99 & 1.45 & $\mathrm{a} / \mathrm{A}$ & 434.60 & 2.22 & $h / C$ \\
\hline \multicolumn{25}{|c|}{ Wine: Graciano (G) } \\
\hline 16 & 1090.42 & 16.68 & $\mathrm{a} / \mathrm{A}$ & 35.25 & 0.46 & $a / B$ & .92 & 2.33 & $a / A$ & 72.85 & 0.02 & $\mathrm{ab} / \mathrm{A}$ & 01 & 0.22 & $\mathrm{cde} / \mathrm{B}$ & 1397.59 & 18.56 & $\mathrm{a} / \mathrm{A}$ & 36 & 0.24 & $\mathrm{~b} / \mathrm{A}$ & 46 & 32 & /A \\
\hline 20 & 1035.55 & 20.86 & $\mathrm{~b} / \mathrm{A}$ & 36.01 & 0.84 & $a / B$ & 262.65 & 5.54 & $\mathrm{a} / \mathrm{A}$ & 71.19 & 0.58 & $\mathrm{abc} / \mathrm{A}$ & 58.73 & 0.88 & cde/A & 1334.20 & 27.24 & $\mathrm{~b} / \mathrm{A}$ & 129.92 & 0.30 & $\mathrm{ab} / \mathrm{A}$ & 1464.13 & 27.54 & $\mathrm{~b} / \mathrm{A}$ \\
\hline 22 & 1002.20 & 4.27 & $\mathrm{~b} / \mathrm{A}$ & 35.76 & 0.29 & $\mathrm{a} / \mathrm{A}$ & 249.08 & 1.08 & $\mathrm{~b} / \mathrm{A}$ & 70.28 & 1.02 & $\mathrm{abc} / \mathrm{A}$ & 56.74 & 0.09 & $\mathrm{de} / \mathrm{A}$ & 1287.04 & 5.64 & $\mathrm{~b} / \mathrm{A}$ & 127.02 & 0.93 & $\mathrm{bc} / \mathrm{A}$ & 1414.06 & 4.71 & $\mathrm{~b} / \mathrm{A}$ \\
\hline 29 & 942.01 & 11.77 & $\mathrm{c} / \mathrm{A}$ & 36.19 & 1.64 & $\mathrm{a} / \mathrm{A}$ & 239.64 & 3.84 & $\mathrm{~b} / \mathrm{A}$ & 68.50 & 0.52 & $\mathrm{abc} / \mathrm{A}$ & 61.96 & 1.86 & $\mathrm{abc} / \mathrm{A}$ & 1217.83 & 17.25 & $\mathrm{c} / \mathrm{A}$ & 130.46 & 1.34 & $\mathrm{ab} / \mathrm{A}$ & 1348.29 & 18.59 & $c / A$ \\
\hline 39 & 816.53 & 2.42 & $\mathrm{~d} / \mathrm{A}$ & 37.01 & 0.92 & $\mathrm{a} / \mathrm{A}$ & 217.02 & 6.19 & $c / A$ & 72.84 & 2.29 & $\mathrm{ab} / \mathrm{A}$ & 59.44 & 1.60 & bcde/A & 1070.56 & 9.53 & $\mathrm{~d} / \mathrm{A}$ & 132.28 & 3.90 & $\mathrm{ab} / \mathrm{A}$ & 1202.85 & 13.43 & $\mathrm{~d} / \mathrm{A}$ \\
\hline 128 & 779.28 & 14.25 & $\mathrm{~d} / \mathrm{A}$ & 36.92 & 0.65 & $\mathrm{a} / \mathrm{A}$ & 205.93 & 6.67 & $c / A$ & 73.65 & 2.32 & $\mathrm{a} / \mathrm{A}$ & 58.48 & 1.81 & cde/A & 1022.14 & 21.31 & I/A & 132.13 & 1.35 & $\mathrm{ab} / \mathrm{A}$ & 1154.26 & 22.63 & $\mathrm{~d} / \mathrm{A}$ \\
\hline 219 & 603.73 & 36.89 & $\mathrm{e} / \mathrm{A}$ & 34.69 & 1.59 & $\mathrm{a} / \mathrm{A}$ & 158.93 & 5.27 & $\mathrm{~d} / \mathrm{B}$ & 65.35 & 3.29 & $\mathrm{c} / \mathrm{A}$ & 55.00 & 2.33 & $\mathrm{e} / \mathrm{A}$ & 797.34 & 43.75 & $\mathrm{e} / \mathrm{A}$ & 120.35 & 5.62 & $c / A$ & 917.69 & 49.37 & e/A \\
\hline 413 & 485.76 & 5.23 & $f / A$ & 35.64 & 0.53 & $\mathrm{a} / \mathrm{A}$ & 147.39 & 1.62 & $\mathrm{de} / \mathrm{B}$ & 67.37 & 4.10 & $\mathrm{bc} / \mathrm{A}$ & 59.74 & 1.49 & bcde/A & 668.78 & 7.22 & $\mathrm{f} / \mathrm{A}$ & 127.11 & 3.16 & $\mathrm{bc} / \mathrm{A}$ & 795.90 & 9.89 & $\mathrm{f} / \mathrm{A}$ \\
\hline 460 & 457.48 & 5.80 & $\mathrm{f} / \mathrm{A}$ & 3510 & 0.24 & $\mathrm{a} / \mathrm{A}$ & 14178 & 2.97 & e/A & 70.41 & 1.27 & $\mathrm{abc} / \mathrm{A}$ & 63.68 & 0.99 & $\mathrm{ab} / \mathrm{A}$ & 634.36 & 8.63 & $\mathrm{f} / \mathrm{A}$ & 134.09 & 1.85 & $\mathrm{ab} / \mathrm{A}$ & 768.45 & 10.48 & $\mathrm{f} / \mathrm{A}$ \\
\hline 602 & 346.45 & 2.94 & $\mathrm{~g} / \mathrm{A}$ & 29.55 & 1.01 & $\mathrm{~b} / \mathrm{A}$ & 134.68 & 3.47 & ef/A & 67.89 & 1.04 & $\mathrm{abc} / \mathrm{A}$ & 63.68 & 1.23 & $\mathrm{ab} /$ & 510.68 & 7.30 & $\mathrm{~g} / \mathrm{A}$ & 131.57 & 0.43 & $\mathrm{ab} / \mathrm{A}$ & 642.25 & 6.99 & $\mathrm{~g} / \mathrm{A}$ \\
\hline 736 & 277.19 & 6.83 & $\mathrm{~h} / \mathrm{A}$ & 30.61 & 1.35 & $\mathrm{~b} / \mathrm{A}$ & 99 & 0.60 & $\mathrm{fg} / \mathrm{A}$ & 72.17 & 1.87 & $\mathrm{ab} / \mathrm{A}$ & 65.19 & 1.58 & $\mathrm{a} / \mathrm{A}$ & 432.79 & 8.10 & $\mathrm{~h} / \mathrm{A}$ & 137.36 & 2.12 & $\mathrm{a} / \mathrm{A}$ & 570.15 & 10.18 & $\mathrm{~h} / \mathrm{A}$ \\
\hline 827 & 230.37 & 4.65 & $\mathrm{i} / \mathrm{A}$ & 29.76 & 0.69 & $\mathrm{~b} / \mathrm{A}$ & 120.64 & 6.57 & $g / A$ & 70.75 & 2.61 & $a b c / A$ & 61.45 & 3.16 & $\mathrm{abcd} / \mathrm{A}$ & 380.77 & 11.89 & $\mathrm{~h} / \mathrm{A}$ & 132.20 & 4.98 & $\mathrm{ab} / \mathrm{A}$ & 512.97 & 16.11 & $h / A$ \\
\hline \multicolumn{25}{|c|}{ Wine: Blend of grapes (M) } \\
\hline 16 & 1075.45 & 0.94 & $\mathrm{a} / \mathrm{A}$ & 37.75 & 1.53 & $\mathrm{a} / \mathrm{A}$ & 230.47 & 2.26 & $a / B$ & 66.11 & 0.28 & $\mathrm{a} / \mathrm{l}$ & 58.94 & 1.62 & $\mathrm{abc} / \mathrm{B}$ & 1343.66 & 0.21 & $a / B$ & 125.05 & 1.89 & $a b c / B$ & 1468.71 & 2.10 & $a / B$ \\
\hline 20 & 99938 & 0.47 & $\mathrm{ab} / \mathrm{B}$ & 36.66 & 0.49 & $a b / B$ & 207.91 & 3.95 & $\mathrm{ab} / \mathrm{B}$ & 64.06 & 1.70 & $a b c / 1$ & 56.68 & 2.02 & $a b c d / A$ & 1243.95 & 4.90 & $\mathrm{~b} / \mathrm{B}$ & 120.75 & 3.73 & $a b c d / B$ & 1364.69 & 1.18 & $a b / B$ \\
\hline 22 & 938.13 & 6.82 & $\mathrm{bc} / \mathrm{B}$ & 36.03 & 0.35 & $a b / A$ & 192.34 & 0.42 & $\mathrm{bc} / \mathrm{B}$ & 64.57 & 0.22 & $\mathrm{ab} /$ & 53.30 & 0.01 & $\mathrm{~cd} / \mathrm{B}$ & 1166.50 & 6.89 & $\mathrm{bc} / \mathrm{B}$ & 117.87 & 0.21 & $\mathrm{bcd} / \mathrm{C}$ & 1284.37 & 6.68 & $\mathrm{bc} / \mathrm{B}$ \\
\hline 29 & 890.21 & 1.48 & $\mathrm{c} / \mathrm{B}$ & 35.09 & 0.42 & $a b / A$ & 189.13 & 3.72 & $\mathrm{bc} / \mathrm{B}$ & 60.61 & 0.45 & $\mathrm{bc} / \mathrm{B}$ & 54.84 & 0.73 & $\mathrm{abcd} / \mathrm{B}$ & 1114.44 & 5.62 & $\mathrm{c} / \mathrm{B}$ & 115.45 & 0.29 & $\mathrm{~d} / \mathrm{C}$ & 1229.89 & 5.33 & $c / B$ \\
\hline 39 & 788.41 & 76.30 & $\mathrm{~d} / \mathrm{AB}$ & 38.2 & 2.17 & $a / A$ & & 13.73 & $c / B$ & 63. & 1.27 & & 54.12 & 4.10 & & 1007.82 & 92.16 & $\mathrm{~d} / \mathrm{AB}$ & 117.20 & 5.27 & & 1125.02 & 97.41 & $d / A B$ \\
\hline 128 & 763.48 & 13.51 & $\mathrm{~d} / \mathrm{AB}$ & 37.50 & 0.47 & $a b / A$ & 179.55 & 16.87 & $\mathrm{c} / \mathrm{AB}$ & 64.14 & 0.15 & $\mathrm{abc} / \mathrm{B}$ & 55.17 & 1.19 & $\mathrm{abcd} / \mathrm{A}$ & 980.54 & 30.36 & $\mathrm{~d} / \mathrm{AB}$ & 119.31 & 1.09 & $\mathrm{abcd} / \mathrm{B}$ & 1099.85 & 31.43 & $\mathrm{~d} / \mathrm{AB}$ \\
\hline 219 & 601.01 & 40.69 & $\mathrm{e} / \mathrm{A}$ & 36.06 & 2.21 & $\mathrm{ab} /$ & 136.19 & 10.57 & $\mathrm{~d} / \mathrm{A}$ & 63.52 & 1.50 & $\mathrm{abc} / \mathrm{A}$ & 52.23 & 2.40 & de/ & 773.25 & 53.47 & $\mathrm{e} / \mathrm{A}$ & 115.75 & 3.90 & $\mathrm{~d} / \mathrm{A}$ & 889.00 & 57.37 & e/A \\
\hline 413 & 464.95 & 14.41 & $\mathrm{f} / \mathrm{AB}$ & 35.84 & 0.77 & $\mathrm{ab}$ & & 5.70 & de/ & 60. & 1.09 & $\mathrm{c} / \mathrm{A}$ & 56.27 & 1.67 & & 626.22 & 19.98 & $\mathrm{f} / \mathrm{AB}$ & 116.39 & 1.87 & & 742.61 & 21.48 & $\mathrm{f} / \mathrm{AB}$ \\
\hline 460 & 440.65 & 8.89 & $\mathrm{f} / \mathrm{AB}$ & 34.19 & 0.70 & $\mathrm{bc} / \mathrm{A}$ & 132.66 & 8.35 & $\mathrm{de} / \mathrm{A}$ & 65.46 & 2.46 & $\mathrm{a} / \mathrm{B}$ & 58.85 & 3.92 & $\mathrm{abcd} / \mathrm{A}$ & 607.50 & 17.38 & $\mathrm{f} / \mathrm{A}$ & 124.31 & 5.45 & $\mathrm{abcd} / \mathrm{B}$ & 731.82 & 22.71 & $\mathrm{f} / \mathrm{A}$ \\
\hline 602 & 322.03 & 2.74 & $\mathrm{~g} / \mathrm{B}$ & 29.85 & 0.43 & $\mathrm{~d} / \mathrm{A}$ & 119.95 & 4.44 & $\mathrm{de} / \mathrm{B}$ & 65.72 & 1.49 & $\mathrm{a} / \mathrm{A}$ & 60.56 & 2.71 & $a b / A$ & 471.84 & 7.11 & $\mathrm{~g} / \mathrm{B}$ & 126.28 & 1.48 & $\mathrm{ab} / \mathrm{B}$ & 598.12 & 6.88 & $\mathrm{~g} / \mathrm{B}$ \\
\hline 736 & 257.69 & 3.38 & $\mathrm{gh} / \mathrm{B}$ & 31.62 & 0.87 & $\mathrm{~cd} / \mathrm{A}$ & 110.53 & 7.55 & $\mathrm{e} / \mathrm{B}$ & 66.44 & 0.95 & $\mathrm{a} / \mathrm{A}$ & 57.76 & 1.33 & abcd/B & 399.84 & 9.56 & $\mathrm{gh} / \mathrm{B}$ & 124.20 & 0.57 & $\mathrm{abcd} / \mathrm{B}$ & 524.04 & 9.18 & $\mathrm{gh} / \mathrm{B}$ \\
\hline 827 & 209.77 & 4.37 & $\mathrm{~h} / \mathrm{B}$ & 29.29 & 0.77 & $\mathrm{~d} / \mathrm{AB}$ & 111.29 & 1.21 & $\mathrm{e} / \mathrm{A}$ & 66.81 & 2.83 & $\mathrm{a} / \mathrm{A}$ & 60.81 & 1.69 & $\mathrm{a} / \mathrm{A}$ & 350.35 & 6.32 & $\mathrm{~h} / \mathrm{B}$ & 127.62 & 4.46 & $\mathrm{a} / \mathrm{A}$ & 477.97 & 10.50 & $\mathrm{~h} / \mathrm{B}$ \\
\hline
\end{tabular}




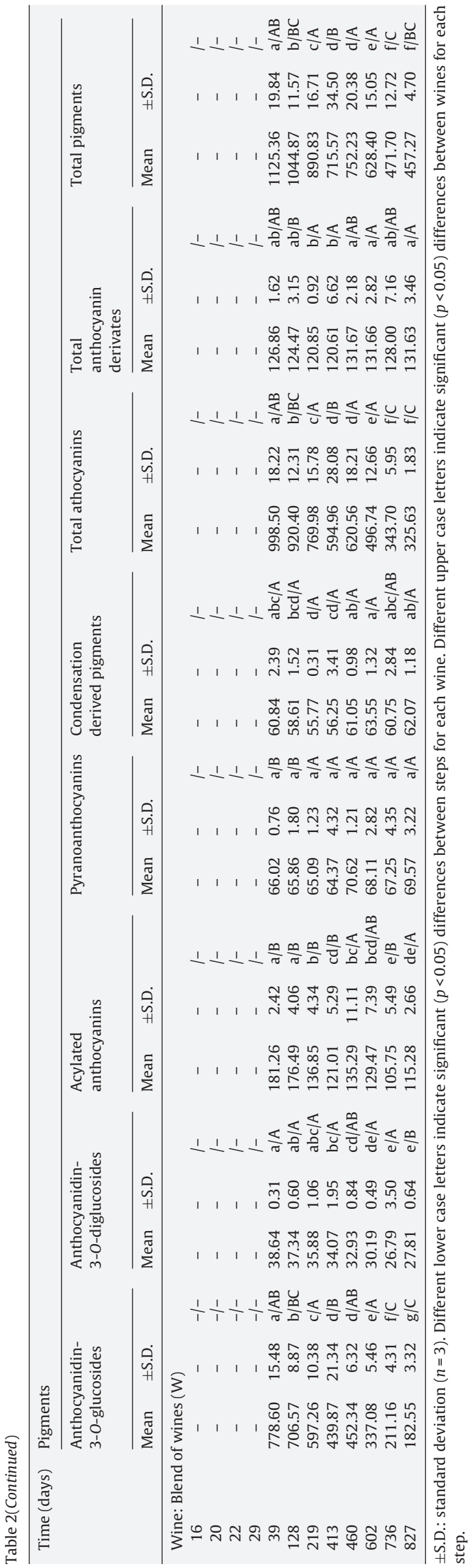

\subsection{ESI-ITMS conditions}

MS analyses were performed using a Finnigan LCQ MS detector (Thermoquest) equipped with an API source, using an electrospray ionization (ESI) interface. The HPLC system was connected to the probe of the mass spectrometer via the UV cell outlet. Both the sheath and the auxiliary gas was nitrogen. The sheath gas flow was $1.2 \mathrm{~L} \mathrm{~min}^{-1}$ and the auxiliary gas flow $6 \mathrm{~L} \mathrm{~min}^{-1}$. The source voltage and the capillary voltage were $4.50 \mathrm{kV}$ and $26 \mathrm{~V}$, respectively, and the capillary temperature $195^{\circ} \mathrm{C}$. Spectra were recorded in positive ion mode between $\mathrm{m} / \mathrm{z} 150$ and 2000 . The mass spectrometer was programmed to do a series of consecutive scans: a full mass and a $\mathrm{MS}^{2}$ scan of the most abundant ion in the full mass. The normalized energy of collision was $45 \%$.

\subsection{Colorimetric measurements}

The absorption spectra were recorded using a Hewlett-Packard UV-visible HP 8453 spectrophotometer (Waldbronn, Germany), employing $2 \mathrm{~mm}$ path length quartz cells. The whole visible spectrum (380-770 nm) was recorded $(\Delta \lambda=1 \mathrm{~nm})$, and Illuminant D65 and $10^{\circ}$ observer were used in the calculations. The CIELAB parameters $\left(L^{*}, a^{*}, b^{*}, C_{\mathrm{ab}}^{*}, h_{\mathrm{ab}}\right)$ and a CIELUV parameter $\left(s_{\mathrm{uv}}^{*}\right)$ were determined by using the original software CromaLab [18], following the recommendations of the 'Commission Internationale de L'Éclairage' [19]. Saturation, $s_{\mathrm{uv}}^{*}$, was included in the colorimetric analysis because it is considered the best correlation for the visually perceived saturation [20].

\subsection{Statistical treatment}

Significant differences were determined by one-way analysis of variance (ANOVA) and Tukey's honestly significant difference test using an SPSS Program, version 13.0 for Windows software package (SPSS Inc., Chicago, IL). Principal component analysis (PCA) was performed using the same SPSS program. This technique was applied to the total correlation matrix. The number of original variables was 73 including the CIELAB and CIELUV parameters $\left(L^{*}, a^{*}, b^{*}, C_{\mathrm{ab}}^{*}, h_{\mathrm{ab}}\right.$ and $\left.s_{\mathrm{uv}}^{*}\right)$, the $\mathrm{pH}$ value of the wine sample, the content of the 46 individual pigments identified (Table 1), and these compounds gathered into different pigment families. The pigment families considered were: the content of anthocyanin monoglucosides (pigments 9, 11, 13, 16 and 18 in Table 1), anthocyanin diglucosides (pigments 2, 4, 5, 10 and 14 in Table 1), anthocyanin-acetyl-glucosides (pigments 19, 24, 30 and 32 in Table 1), anthocyanin-caffeoyl-glucosides (pigment 43 in Table 1), anthocyanin-p-coumaroyl-glucosides (pigments 33, 34, 36-38, 40 and 41 in Table 1), total anthocyaninacyl-glucosides (the sum of anthocyanin-acetyl-glucosides plus anthocyanin-caffeoyl-glucosides plus anthocyanin-p-coumaroylglucosides), total anthocyanins (the sum of anthocyanin monoglucosides, diglucosides and acyl-glucosides), anthocyanin-flavanol acetaldehyde-mediated condensation products (pigments 23,25 , 26, 28 and 29 in Table 1), direct flavanol-anthocyanin condesation products (pigments 1, 3, 7 and 8 in Table 1), total condensation products (the sum of the previous two families), A-type vitisins (pigments 6, 12, 15, 17 and 31 in Table 1), B-type vitisins (pigment 21 in Table 1), total vitisins (the sum of A-type vitisins plus B-type vitisins), 4-vinylphenol anthocyanins (pigments 42, 44 and 45 in Table 1) 4-vinylcatechol anthocyanins (pigment 39 in Table 1), 4-vinylguaiacol anthocyanins (pigments 43 and 46 in Table 1), total vinyl-anthocyanin adducts (the sum of 4-vinylphenol anthocyanins plus 4-vinylcatechol anthocyanins plus 4-vinylguaiacol anthocyanins), total pyranoanthocyanins (the sum of total vitisins plus total vinyl-anthocyanin adducts), total derived pigments (the sum of total condesation products plus total pyranoanthocyanins) 
Colour

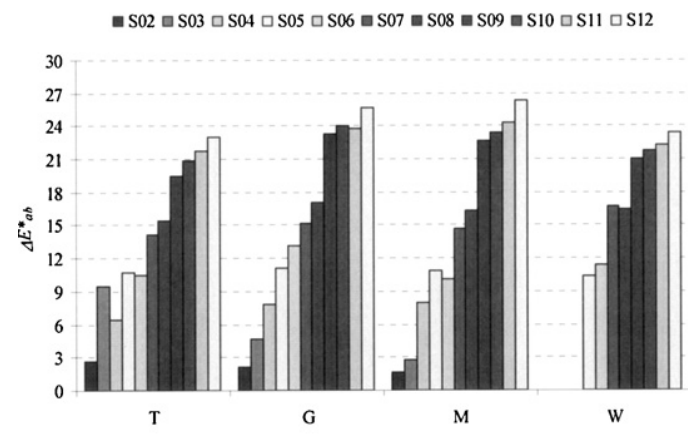

Colour

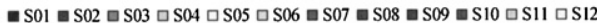

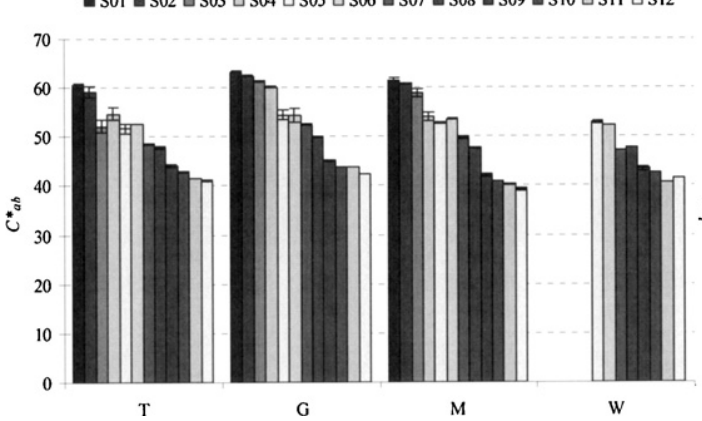

Colour

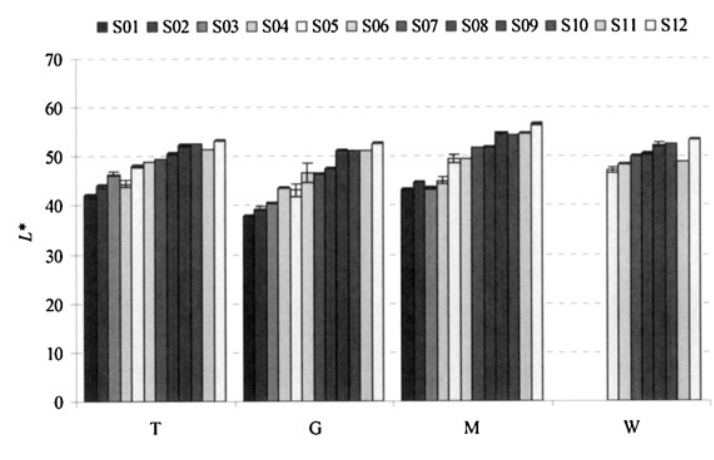

Colour

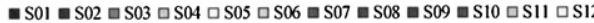

$$
12
$$

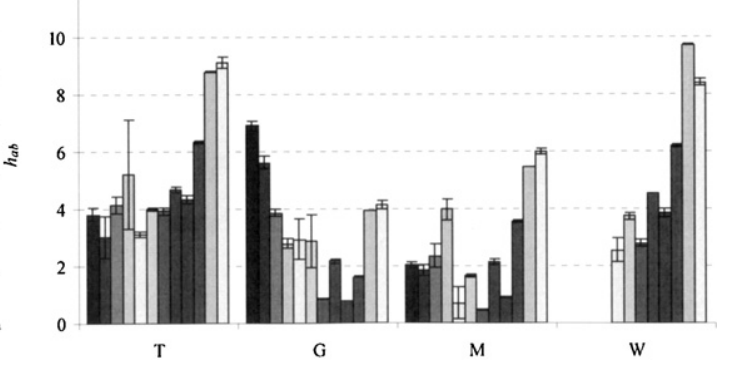

Fig. 1. Evolution of colour parameters: colour differences $\left(\Delta E_{\mathrm{ab}}^{*}\right)$, lightness $\left(L^{*}\right)$, chroma $\left(C_{\mathrm{ab}}^{*}\right)$ and hue $\left(h_{\mathrm{ab}}\right)$ of $\mathrm{T}, \mathrm{G}, \mathrm{M}$ and $\mathrm{W}$ wines during winemaking.

Table 3

Summary of the total correlation table in which correlation coefficients between colour parameters $\left(L^{*}, C_{\mathrm{ab}}^{*}, h_{\mathrm{ab}}, s_{\mathrm{uv}}^{*}, a^{*}\right.$ and $\left.b^{*}\right)$ and main anthocyanin pigments.

\begin{tabular}{|c|c|c|c|c|c|c|c|c|c|c|c|c|c|}
\hline \multirow[t]{2}{*}{ Colour parameters } & \multicolumn{13}{|c|}{ Anthocyanin pigments } \\
\hline & 1 & 2 & 3 & 4 & 5 & 6 & 7 & 8 & 9 & 10 & 11 & 12 & 13 \\
\hline \multicolumn{14}{|c|}{ Wine: Tempranillo (T) } \\
\hline$L^{*}$ & $-0.945^{* *}$ & $-0.913^{* *}$ & $-0.953^{* *}$ & $-0.945^{* *}$ & $-0.954^{* *}$ & $-0.952^{* *}$ & $-0.786^{* *}$ & $-0.938^{* *}$ & $-0.590^{*}$ & $-0.943^{* *}$ & $-0.949^{* *}$ & $-0.954^{* *}$ & $-0.955^{* *}$ \\
\hline$C_{\mathrm{ab}}^{*}$ & $0.976^{* *}$ & $0.942^{* *}$ & $0.978^{* *}$ & $0.962^{* *}$ & $0.978^{* *}$ & $0.978^{* *}$ & $0.879^{* *}$ & $0.968^{* *}$ & $0.716^{* *}$ & $0.939^{* *}$ & $0.960^{* *}$ & $0.979^{* *}$ & $0.979^{* *}$ \\
\hline$h_{\mathrm{ab}}^{\mathrm{db}}$ & $-0.808^{* *}$ & $-0.836^{* *}$ & $-0.797^{* *}$ & $-0.789^{* *}$ & $-0.792^{* *}$ & $-0.797^{* *}$ & $-0.885^{* *}$ & $-0.747^{* *}$ & $-0.731^{* *}$ & $-0.641^{*}$ & $-0.693^{*}$ & $-0.791^{* *}$ & $-0.790^{* *}$ \\
\hline$s_{\mathrm{uv}}^{*}$ & $0.938^{* *}$ & $0.883^{* *}$ & $0.942^{* *}$ & $0.924^{* *}$ & $0.943^{* *}$ & $0.941^{* *}$ & $0.760^{* *}$ & $0.957^{* *}$ & $0.657^{*}$ & $0.955^{* *}$ & $0.965^{* *}$ & $0.945^{* *}$ & $0.946^{* *}$ \\
\hline$a^{*}$ & $0.978^{* *}$ & $0.946^{* *}$ & $0.980^{* *}$ & $0.964^{* *}$ & $0.979^{* * *}$ & $0.979^{* *}$ & $0.884^{* *}$ & $0.969^{* *}$ & $0.721^{* *}$ & $0.937^{* *}$ & $0.960^{* *}$ & $0.981^{* *}$ & $0.981^{* *}$ \\
\hline$b^{*}$ & $-0.636^{*}$ & $-0.688^{*}$ & $-0.621^{*}$ & $-0.618^{*}$ & $-0.616^{*}$ & $-0.622^{*}$ & $-0.767^{* *}$ & -0.567 & $-0.660^{*}$ & -0.460 & -0.512 & $-0.616^{*}$ & $-0.614^{*}$ \\
\hline \multicolumn{14}{|l|}{ Wine: Graciano (G) } \\
\hline$L^{*}$ & $-0.959^{* *}$ & $-0.926^{* *}$ & $-0.963^{* *}$ & $-0.973^{* *}$ & $-0.968^{* *}$ & $-0.967^{* *}$ & $-0.688^{*}$ & $-0.947^{* *}$ & $-0.840^{* *}$ & $-0.968^{* *}$ & $-0.965^{* *}$ & $-0.968^{* *}$ & $-0.967^{* *}$ \\
\hline$C_{\mathrm{ab}}^{*}$ & $0.979^{* *}$ & $0.941^{* *}$ & $0.985^{* *}$ & $0.987^{* *}$ & $0.987^{* *}$ & $0.986^{* *}$ & $0.738^{* *}$ & $0.971^{* *}$ & $0.904^{* *}$ & $0.969^{* *}$ & $0.975^{* *}$ & $0.986^{* *}$ & $0.985^{* *}$ \\
\hline$h_{\mathrm{ab}}^{\mathrm{ab}}$ & 0.458 & 0.402 & 0.477 & 0.548 & 0.515 & 0.503 & 0.047 & 0.545 & 0.389 & $0.631^{*}$ & $0.602^{*}$ & 0.516 & 0.521 \\
\hline$s_{\mathrm{uv}}^{*}$ & $0.932^{* *}$ & $0.878^{* *}$ & $0.941^{* *}$ & $0.967^{* *}$ & $0.955^{* *}$ & $0.950^{* *}$ & $.595^{*}$ & $0.945^{* *}$ & $0.830^{* *}$ & $0.970^{* *}$ & $0.966^{* *}$ & $0.953^{* *}$ & $0.953^{* *}$ \\
\hline$a^{*}$ & $0.980^{* *}$ & $0.943^{* *}$ & $0.986^{* *}$ & $0.986^{* *}$ & $0.987^{* *}$ & $0.987^{* *}$ & $0.743^{* *}$ & $0.971^{* *}$ & $0.906^{* *}$ & $0.967^{* *}$ & $0.974^{* *}$ & $0.986^{* *}$ & $0.985^{* *}$ \\
\hline$b^{*}$ & $0.632^{*}$ & 0.571 & $0.648^{*}$ & $0.712^{* *}$ & $0.683^{*}$ & $0.671^{*}$ & 0.217 & $0.698^{*}$ & 0.530 & $0.776^{* *}$ & $0.750^{* *}$ & $0.682^{*}$ & $0.686^{*}$ \\
\hline \multicolumn{14}{|c|}{ Wine: Mixture of grapes (M) } \\
\hline$L^{*}$ & $-0.965^{* *}$ & $-0.910^{* *}$ & $-0.968^{* *}$ & $-0.963^{* *}$ & $-0.968^{* *}$ & $-0.968^{* *}$ & $-0.712^{* *}$ & $-0.956^{* *}$ & $-0.816^{* *}$ & $-0.923^{* *}$ & $-0.944^{* *}$ & $-0.966^{* *}$ & $-0.966^{* *}$ \\
\hline$C_{\mathrm{ab}}^{*}$ & $0.980^{* *}$ & $0.935^{* *}$ & $0.980^{* *}$ & $0.981^{* *}$ & $0.983^{* *}$ & $0.983^{* *}$ & $0.823^{* *}$ & $0.930^{* *}$ & $0.853^{* *}$ & $0.943^{* *}$ & $0.954^{* *}$ & $0.981^{* *}$ & $0.981^{* *}$ \\
\hline$h_{\mathrm{ab}}^{\mathrm{ab}}$ & -0.516 & $-0.630^{*}$ & -0.503 & -0.482 & -0.488 & -0.497 & $-0.778^{* *}$ & -0.365 & -0.247 & -0.419 & -0.405 & -0.491 & -0.487 \\
\hline$s_{\mathrm{uv}}^{*}$ & $0.951^{* *}$ & $0.878^{* *}$ & $0.955^{* *}$ & $0.965^{* *}$ & $0.963^{* *}$ & $0.960^{* *}$ & $0.692^{*}$ & $0.952^{* *}$ & $0.891^{* *}$ & $0.935^{* *}$ & $0.955^{* *}$ & $0.961^{* *}$ & $0.962^{* *}$ \\
\hline$a^{*}$ & $0.980^{* *}$ & $0.936^{* *}$ & $0.980^{* *}$ & $0.981^{* *}$ & $0.983^{* *}$ & $0.983^{* *}$ & $0.826^{* *}$ & $0.929^{* *}$ & $0.851^{* *}$ & $0.943^{* *}$ & $0.953^{* *}$ & $0.981^{* *}$ & $0.981^{* *}$ \\
\hline$b^{*}$ & -0.265 & -0.400 & -0.251 & -0.235 & -0.238 & -0.247 & $-0.616^{*}$ & -0.104 & -0.040 & -0.185 & -0.161 & -0.241 & -0.237 \\
\hline \multicolumn{14}{|c|}{ Wine: Blend of wines (W) } \\
\hline$L^{*}$ & -0.661 & -0.651 & -0.681 & -0.618 & -0.689 & -0.681 & -0.592 & -0.469 & -0.676 & -0.654 & -0.618 & -0.678 & -0.674 \\
\hline$C_{\mathrm{ab}}^{*}$ & $0.943^{* *}$ & $0.926^{* *}$ & $0.945^{* *}$ & $0.943^{* *}$ & $0.943^{* *}$ & $0.945^{* *}$ & $0.946^{* *}$ & $0.825^{*}$ & $0.872^{* *}$ & $0.902^{* *}$ & $0.898^{* *}$ & $0.947^{* *}$ & $0.945^{* *}$ \\
\hline$h_{\mathrm{ab}}^{\mathrm{ab}}$ & $-0.914^{* *}$ & $-0.922^{* *}$ & $-0.904^{* *}$ & $-0.919^{* *}$ & $-0.895^{* *}$ & $-0.901^{* *}$ & $-0.945^{* *}$ & $-0.880^{* *}$ & -0.552 & -0.687 & $-0.748^{*}$ & $-0.892^{* *}$ & $-0.891^{* *}$ \\
\hline$S_{\mathrm{uv}}^{*}$ & $0.889^{* *}$ & $0.868^{* *}$ & $0.898^{* *}$ & $0.883^{* *}$ & $0.900^{* *}$ & $0.899^{* *}$ & $0.866^{* *}$ & $0.759^{*}$ & $0.908^{* *}$ & $0.907^{* *}$ & $0.885^{* *}$ & $0.904^{* *}$ & $0.902^{* *}$ \\
\hline$a^{*}$ & $0.949^{* *}$ & $0.933^{* *}$ & $0.951^{* *}$ & $0.950^{* *}$ & $0.948^{* *}$ & $0.951^{* *}$ & $0.954^{* *}$ & $0.835^{* *}$ & $0.866^{* *}$ & $0.900^{* *}$ & $0.900^{* *}$ & $0.953^{* *}$ & $0.950^{* *}$ \\
\hline$b^{*}$ & $-0.882^{* *}$ & $-0.893^{* *}$ & $-0.872^{* *}$ & $-0.890^{* *}$ & $-0.863^{* *}$ & $-0.869^{* *}$ & $-0.912^{* *}$ & $-0.865^{* *}$ & -0.495 & -0.636 & -0.706 & $-0.859^{* *}$ & $-0.858^{* *}$ \\
\hline
\end{tabular}

Anthocyanin pigments: (1) delphinidin-3-O-glucoside, (2) cyanidin-3-O-glucoside, (3) petunidin-3-O-glucoside, (4) peonidin-3-O-glucoside, (5) malvidin-3-O-glucoside, (6) anthocyanin monoglucosides, (7) anthocyanin diglucosides, (8) anthocyanin-acetyl-glucosides, (9) anthocyanin-caffeoyl-glucosides, (10) anthocyanin-p-coumaroylglucosides, (11) total anthocyanin-acyl-glucosides, (12) total anthocyanins, (13) total pigments.

* Pearson's correlations are significant at the 0.05 level (two-tailed).

** Pearson's correlations are significant at the 0.01 level (two-tailed). 
Table 4

Summary of the total correlation table in which correlation coefficients between colour parameters $\left(L^{*}, C_{\mathrm{ab}}^{*}, h_{\mathrm{ab}}, s_{\mathrm{uv}}^{*}, a^{*}\right.$ and $\left.b^{*}\right)$ and main anthocyanin derived pigments.

\begin{tabular}{|c|c|c|c|c|c|c|c|c|c|c|c|c|}
\hline \multirow[t]{2}{*}{ Colour parameters } & \multicolumn{12}{|c|}{ Anthocyanin derived pigments } \\
\hline & 1 & 2 & 3 & 4 & 5 & 6 & 7 & 8 & 9 & 10 & 11 & 12 \\
\hline \multicolumn{13}{|c|}{ Wine: Tempranillo ( $\mathrm{T}$ ) } \\
\hline$L^{*}$ & 0.258 & -0.056 & 0.249 & -0.414 & $-0.655^{*}$ & -0.553 & $0.799^{* *}$ & $0.782^{* *}$ & $0.790^{* *}$ & $0.802^{* *}$ & 0.482 & 0.435 \\
\hline$C_{\mathrm{ab}}^{*}$ & -0.377 & 0.252 & -0.279 & 0.442 & $0.606^{*}$ & 0.572 & $-0.859^{* *}$ & $-0.863^{* *}$ & $-0.848^{* *}$ & $-0.867^{* *}$ & -0.560 & -0.496 \\
\hline$h_{\mathrm{ab}}^{\mathrm{ab}}$ & $0.641^{*}$ & $-0.641^{*}$ & 0.369 & $-0.617^{*}$ & -0.134 & $-0.654^{*}$ & $0.834^{* *}$ & $0.924^{* *}$ & $0.832^{* *}$ & $0.866^{* *}$ & 0.439 & 0.516 \\
\hline$s_{\mathrm{uv}}^{*}$ & -0.209 & 0.127 & -0.161 & 0.334 & $0.644^{*}$ & 0.469 & $-0.768^{* *}$ & $-0.751^{* *}$ & $-0.770^{* *}$ & $-0.773^{* *}$ & -0.558 & -0.396 \\
\hline$a^{*}$ & -0.385 & 0.264 & -0.282 & 0.449 & $0.598^{*}$ & $0.577^{*}$ & $-0.863^{* *}$ & $-0.870^{* *}$ & $-0.854^{* *}$ & $-0.873^{* *}$ & -0.561 & -0.499 \\
\hline$b^{*}$ & $0.643^{*}$ & $-0.683^{*}$ & 0.351 & -0.563 & 0.061 & -0.560 & $0.684^{*}$ & $0.809^{* *}$ & $0.687^{*}$ & $0.725^{* *}$ & 0.349 & 0.458 \\
\hline \multicolumn{13}{|l|}{ Wine: Graciano (G) } \\
\hline$L^{*}$ & 0.562 & 0.540 & $0.645^{*}$ & $-0.768^{* *}$ & -0.566 & $-0.770^{* *}$ & $0.844^{* *}$ & $0.848^{* *}$ & $0.848^{* *}$ & $0.874^{* *}$ & -0.239 & 0.324 \\
\hline$C_{\mathrm{ab}}^{*}$ & -0.564 & -0.541 & $-0.647^{*}$ & $0.748^{* *}$ & 0.556 & $0.751^{* *}$ & $-0.877^{* *}$ & $-0.900^{* *}$ & $-0.848^{* *}$ & $-0.904^{* *}$ & 0.187 & -0.357 \\
\hline$h_{\mathrm{ab}}^{\mathrm{ab}}$ & -0.117 & -0.211 & -0.166 & $0.642^{*}$ & 0.246 & $0.603^{*}$ & -0.178 & -0.178 & -0.310 & -0.223 & $0.601^{*}$ & 0.241 \\
\hline$s_{\mathrm{uv}}^{*}$ & -0.485 & -0.515 & -0.572 & $0.781^{* *}$ & 0.536 & $0.776^{* *}$ & $-0.803^{* *}$ & $-0.785^{* *}$ & $-0.843^{* *}$ & $-0.837^{* *}$ & 0.281 & -0.246 \\
\hline$a^{*}$ & -0.567 & -0.542 & $-0.650^{*}$ & $0.744^{* *}$ & 0.557 & $0.748^{* *}$ & $-0.880^{* *}$ & $-0.904^{* *}$ & $-0.849^{* *}$ & $-0.907^{* *}$ & 0.181 & -0.362 \\
\hline$b^{*}$ & -0.230 & -0.300 & -0.289 & $0.731^{* *}$ & 0.362 & $0.701^{*}$ & -0.390 & -0.371 & -0.507 & -0.432 & 0.545 & 0.117 \\
\hline \multicolumn{13}{|c|}{ Wine: Blend of grapes (M) } \\
\hline$L^{*}$ & 0.299 & $0.651^{*}$ & 0.462 & $-0.613^{*}$ & $-0.648^{*}$ & $-0.708^{*}$ & $0.857^{* *}$ & $0.807^{* *}$ & $0.868^{* *}$ & $0.871^{* *}$ & 0.308 & 0.442 \\
\hline$C_{\mathrm{ab}}^{*}$ & -0.374 & $-0.591^{*}$ & -0.514 & $0.650^{*}$ & $0.654^{*}$ & $0.743^{* *}$ & $-0.867^{* *}$ & $-0.880^{* *}$ & $-0.878^{* *}$ & $-0.895^{* *}$ & -0.303 & -0.472 \\
\hline$h_{\mathrm{ab}}^{\mathrm{ab}}$ & 0.573 & 0.065 & 0.543 & -0.389 & -0.278 & -0.423 & $0.658^{*}$ & $0.628^{*}$ & $0.606^{*}$ & $0.654^{*}$ & 0.330 & 0.504 \\
\hline$s_{\mathrm{uv}}^{*}$ & -0.199 & $-0.643^{*}$ & -0.369 & $0.696^{*}$ & $0.632^{*}$ & $0.783^{* *}$ & $-0.788^{* *}$ & $-0.780^{* *}$ & $-0.819^{* *}$ & $-0.815^{* *}$ & -0.180 & -0.321 \\
\hline$a^{*}$ & -0.377 & $-0.588^{*}$ & -0.515 & $0.650^{*}$ & $0.655^{*}$ & $0.744^{* *}$ & $-0.869^{* *}$ & $-0.881^{* *}$ & $-0.880^{* *}$ & $-0.897^{* *}$ & -0.304 & -0.474 \\
\hline$b^{*}$ & 0.518 & -0.113 & 0.440 & -0.262 & -0.113 & -0.270 & 0.425 & 0.429 & 0.371 & 0.422 & 0.215 & 0.383 \\
\hline \multicolumn{13}{|c|}{ Wine: Blend of wines (W) } \\
\hline$L^{*}$ & 0.246 & 0.064 & 0.366 & -0.008 & -0.191 & -0.106 & 0.687 & 0.572 & $0.720^{*}$ & 0.695 & 0.662 & 0.530 \\
\hline$C_{\mathrm{ab}}^{*}$ & -0.631 & 0.506 & -0.461 & 0.249 & -0.141 & 0.280 & $-0.752^{*}$ & $-0.865^{* *}$ & -0.600 & $-0.800^{*}$ & -0.639 & -0.575 \\
\hline$h_{\mathrm{ab}}^{\mathrm{ab}}$ & $0.787^{*}$ & $-0.754^{*}$ & 0.486 & -0.676 & 0.670 & -0.618 & $0.770^{*}$ & $0.869^{* *}$ & 0.658 & $0.821^{*}$ & 0.400 & 0.477 \\
\hline$S_{\mathrm{uv}}^{*}$ & -0.479 & 0.334 & -0.385 & 0.141 & 0.026 & 0.212 & $-0.715^{*}$ & $-0.777^{*}$ & -0.621 & $-0.755^{*}$ & -0.644 & -0.533 \\
\hline$a^{*}$ & -0.644 & 0.523 & -0.465 & 0.269 & -0.164 & 0.297 & $-0.759^{*}$ & $-0.872^{* *}$ & -0.606 & $-0.807^{*}$ & -0.634 & -0.575 \\
\hline$b^{*}$ & $0.760^{*}$ & $-0.741^{*}$ & 0.461 & $-0.725^{*}$ & $0.729^{*}$ & -0.657 & $0.743^{*}$ & $0.836^{* *}$ & 0.665 & $0.797^{*}$ & 0.343 & 0.436 \\
\hline
\end{tabular}

Anthocyanin derived pigments: (1) acetaldehyde-mediated F-A condensation products, (2) direct F-A condesation products, (3) total condesation products, (4) A-type vitisins, (5) B-type vitisins, (6) total Vtitisins, (7) 4-vinylphenol anthocyanins, (8) 4-vinylcatechol anthocyanins, (9) 4-vinylguaiacol anthocyanins, (10) total vinyl-anthocyanin adducts, (11) pyranoanthocyanins, (12) total derived pigments.

* Pearson's correlations are significant at the 0.05 level (two-tailed).

** Pearson's correlations are significant at the 0.01 level (two-tailed).

and total pigments (the sum of total anthocyanins plus total derived pigments).

The number of samples used was 132. These samples correspond to 12 steps for wines T, $G$ and $M$ and 8 steps for $W$ wine. All samples were taken in triplicate.

\section{Results and discussion}

\subsection{Composition analysis}

The identification of the pigments was carried out taking into account their chromatographic retention times, UV-vis spectra and molecular and fragment ions supplied by the $\mathrm{M}^{+}$and $\mathrm{MS}^{2}$ analysis [21]. The pigments belong to different families: anthocyanins, acylated anthocyanins, pyranoanthocyanins, direct flavanol-anthocyanin condensation products and acetaldehydemediated flavanol-anthocyanin condensation products. Table 1 shows mass spectral data of the pigments identified in the wine samples. The number of peaks corresponds to their elution order in RP-HPLC.

The use of mass spectrometry coupled to HPLC-DAD has allowed the detection of pigments at low concentrations. Monoglycosides of malvidin and of cyanidin other than glucosides were detected. Wang et al. [22] found malvidin-3-O-galactoside and its acyl derivatives in Cabernet Sauvignon grape skin extracts. Acetylgalactoside anthocyanins were detected in monovarietal wines produced in Uruguay [23] and Valentão et al. [24] found cyanidin3-O-galactoside in wines obtained from Touriga Nacional grapes. Although, as far as we know, there have been no reports of anthocyanidins substituted with other monosaccharides in grapes or wines, compounds 20 and 27 must not be galactosides since their elution (later than their corresponding glucosides) does not coincide with the pattern of elution in reversed-phase columns described for these anthocyanins in the literature [22,25-28].

Table 2 shows the mean concentration $\left(\mathrm{mg} \mathrm{L}^{-1}, \pm\right.$ S.D.; $n=3$ ) of different pigment families during winemaking and ageing of wines T, G, M and W. There were significant differences in total pigments' contents between $\mathrm{T}, \mathrm{G}$ and $\mathrm{M}$ wines from the beginning of the wine-

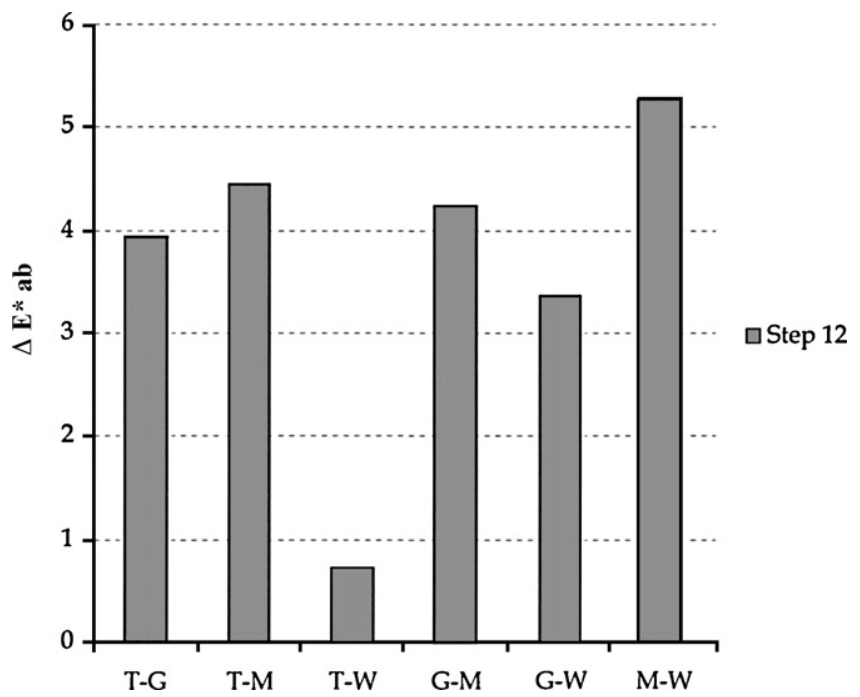

Fig. 2. Colour differences $\left(\Delta E_{\mathrm{ab}}^{*}\right)$ between $\mathrm{T}, \mathrm{G}, \mathrm{M}$ and $\mathrm{W}$ wines at the end of the study. 

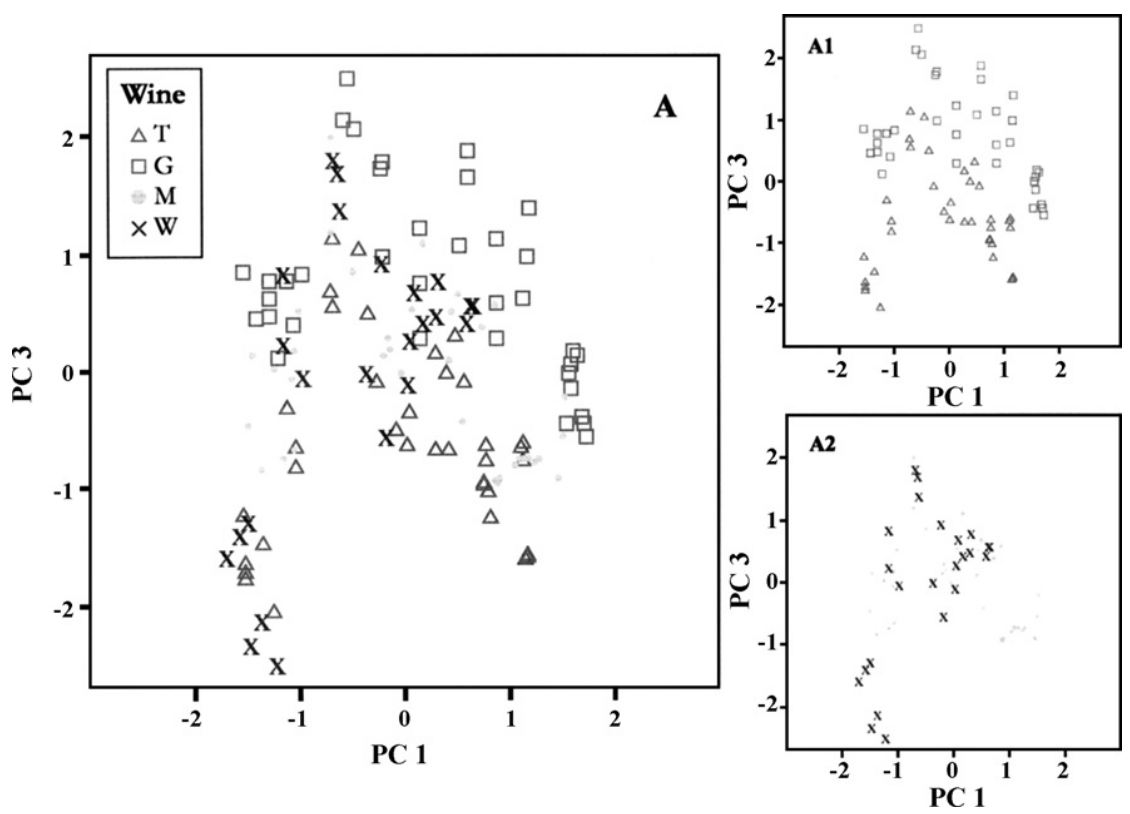

Fig. 3. Scores of the wine samples on the plane defined by the first and third principal components (PC1 and PC3). A: projection of the whole samples (T, G, M and W). A1: projection of $\mathrm{T}$ and $\mathrm{G}$ samples. A2: projection of $\mathrm{M}$ and $\mathrm{W}$ samples.

making and in most of the winemaking steps. The total content of pigments in $\mathrm{M}$ wine after alcoholic fermentation was $1469 \mathrm{mg} \mathrm{L}^{-1}$. This content is slightly higher than the contribution to the total pigment amount that would be expected due to the incorporation of the Graciano variety, that is to say, higher than the sum of the $80 \%$ of total pigments in $\mathrm{T}\left(1104 \mathrm{mg} \mathrm{L}^{-1}\right)$ and the $20 \%$ of total pigments in $\mathrm{G}$ (305 $\mathrm{mg} \mathrm{L}^{-1}$ ), i.e. $1409 \mathrm{mg} \mathrm{L}^{-1}$. On the other hand, and as expected, there was a reduction in the anthocyanidin monoglucosides' content during winemaking. This reduction is slightly greater in T wine (83\%) than in $\mathrm{G}$ and $\mathrm{M}$ wines (79 and $80 \%$, respectively) indicating that these anthocyanins in the $\mathrm{M}$ wine are more protected than in Tempranillo varietal wine. These results are in contrast to those reported by Monagas et al. [11] who showed that the anthocyanin glucosides in the Tempranillo-Graciano blended wines disappeared

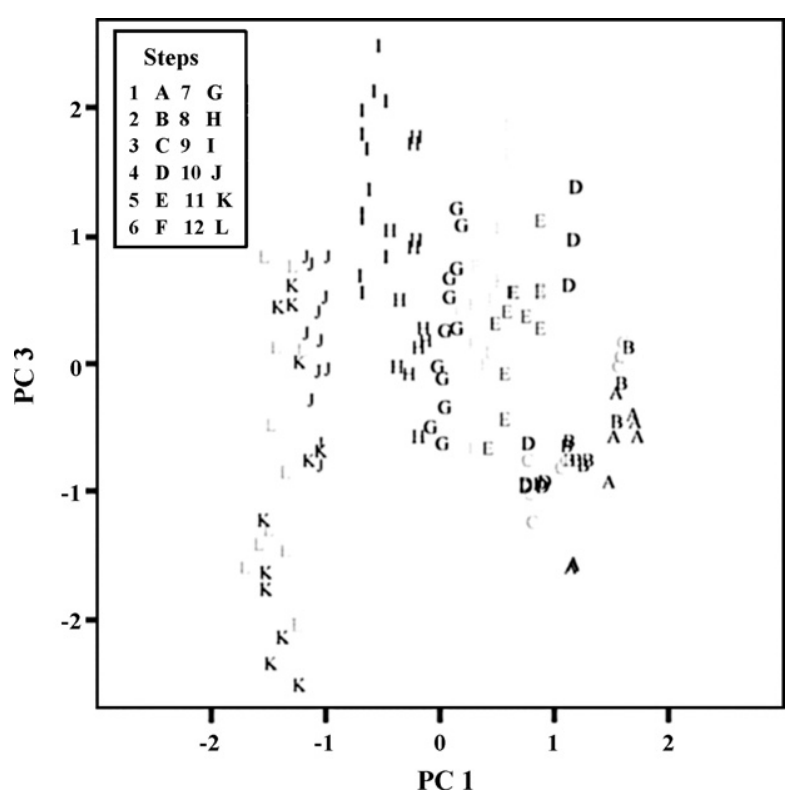

Fig. 4. Scores of the wine samples on the plane defined by the first and third principal components (PC1 and PC3). Samples are coloured in accordance with the winemaking step. faster than in Tempranillo base wine. Nonetheless, they seem to support the views of Boulton [4], who suggested that colour extraction and retention in the wine are not only dependent on the concentration of pigments in the berry, but also on the levels of cofactors. In a recent work carried out in our laboratory [12] we observed that the extent of the interaction between pigments and flavanols was related not only to the concentration of flavanols, but also to the qualitative composition, the flavanols of grape skins of the Graciano variety being better copigments than those of Tempranillo whereas the opposite was found for the flavanols from seeds. Since flavanols from grape skin are more available than those from seeds, it could be said that the flavanols from the skin of the Graciano variety may facilitate the extraction and retention of pigments from the Tempranillo variety.

As far as anthocyanidin diglucosides are concerned, they were present in both Tempranillo and Graciano wines. A number of studies have reported their presence in $V$. vinifera grapes or wines [21,29-31]. The levels of these compounds decrease during winemaking (14-24\%). Although the decrease is not as strong as occurs in monoglucosides. This indicates the greater stability or the lesser reactivity of the diglucosides compared to the monoglucosides. There were significant differences in the contents of anthocyanidin diglucosides between $\mathrm{G}$ and $\mathrm{T}$ wines at the end of the study, but not between these wines and $M$, showing $G$ wine with higher content in this type of compounds.

The decrease in acylated anthocyanins (47-55\%) is lower than in the non-acylated ones. Although at the beginning of the winemaking process the concentration of acylated anthocyanins is higher in $\mathrm{G}$ and $\mathrm{M}$ wines than in $\mathrm{T}$ wine, there were no significant differences between them at the end of the study.

Regarding anthocyanin derived pigments, $M$ wine generally has the lower contents in pigments formed by condensation between anthocyanins and flavanols, although, at the end of the study, the differences between wines were not significant.

\subsection{Colorimetric analysis}

Fig. 1 shows the evolution of colour parameters: lightness, chroma and hue and the $\Delta E_{\mathrm{ab}}^{*}$ of T, G, M and W wines. During winemaking and ageing an important increase in lightness $L^{*}$ occurs 

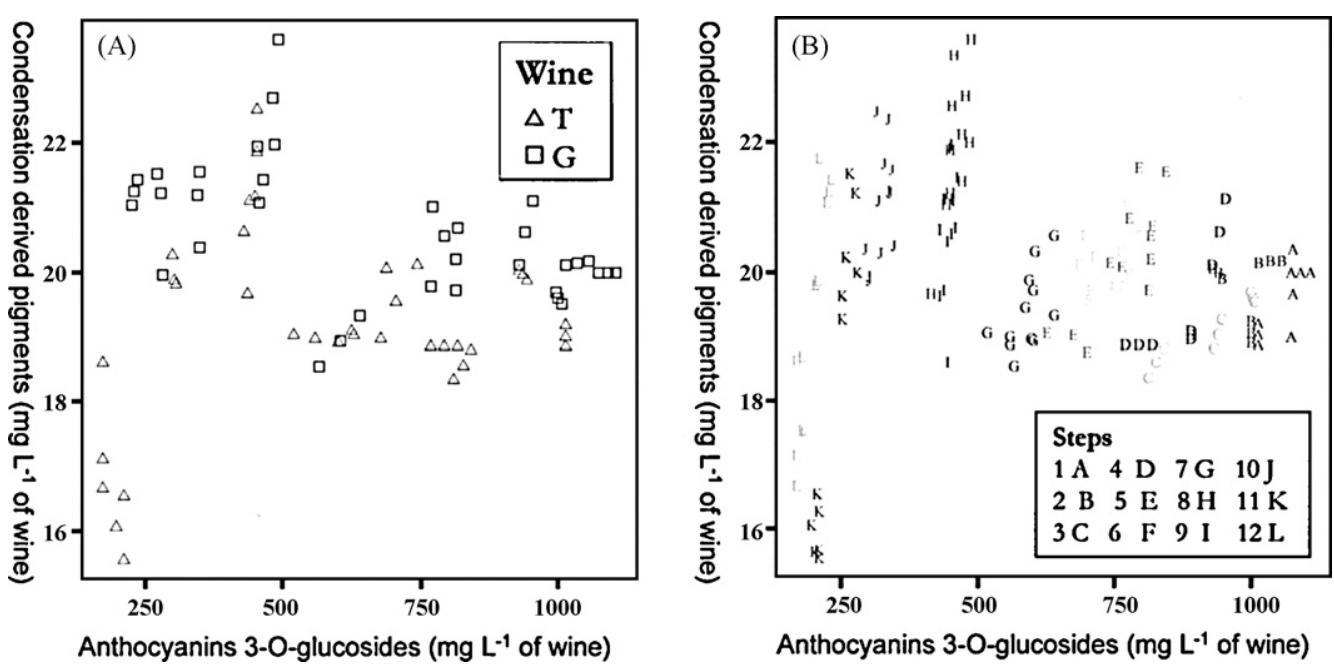

Fig. 5. Projection of the wine samples considering only the first original variables most related to PC1 (anthocyanins 3-glucosides) and PC3 (total condensation products). A: according with the type of wine ( $\mathrm{T}$ or $\mathrm{G}$ wine). B: according with the winemaking step.

which is more noticeable in $\mathbf{G}$ and $\mathbf{M}$ (increases of 39 and 33\%, respectively) than in $\mathbf{T}$ (an increase of $26 \%$ ). Chroma $\left(C_{\mathrm{ab}}^{*}\right)$ and saturation $\left(s_{\mathrm{uv}}^{*}\right)$ decrease in concordance with the fall of anthocyanins. It is worth pointing out that there was a stabilization of the Chroma values in the last samplings of wines which correspond to the end of the maturation in oak barrels and to the ageing in bottles. This was accompanied by changes in the values of the hue, which clearly increases in these latest samples. At the end of the study, relevant differences existed in hue $h_{\mathrm{ab}}$, being higher in $\mathbf{T}$ and $\mathbf{W}$ (more orange-red) than in $\mathbf{G}$ and $\mathbf{M}$ (more blue-red). Colour differences $\left(\Delta E_{\mathrm{ab}}^{*}\right.$ ) between the sample taken after alcoholic fermentation (S01) and the next ones (samples S02-S12) increased along the study, although a slight tendency to the colour stabilization could be observed during maturation in bottle.

In the last step (after 12 months in bottles), colour differences between wines were detectable by the human eye (since $\Delta E_{\mathrm{ab}}^{*}$ value is $\geq 3$ ) in all cases except for $\mathbf{T}$ and $\mathbf{W}$ (Fig. 2). These wines had indistinguishable colours even when using analytical methods $\left(\Delta E_{\mathrm{ab}}^{*} \leq 1\right)$. Results indicate that $\mathbf{M}$ wine was closer to $\mathbf{G}$ wine than to $\mathbf{W}$ wine and that this later was closer to $\mathbf{T}$ wine.

\subsection{Statistical correlations}

Tables 3 and 4 are a summary of the total correlation table in which main correlation coefficients between colour parameters $\left(L^{*}\right.$, $C_{\mathrm{ab}}^{*}, h_{\mathrm{ab}}, s_{\mathrm{uv}}^{*}, a^{*}$ and $\left.b^{*}\right)$ and main anthocyanin and anthocyanin derived pigments, respectively, are given. Statistical correlations show that, on the one hand there is a negative correlation between the $L^{*}$ parameter and anthocyanins (glucosides, diglucosides and acylated anthocyanins) and, on the other, there is a direct correlation between $L^{*}$ and many of the derived pigments (mainly vinyl-anthocyanins). This shows that the decrease of anthocyanins and the increase of the derived pigments, as occurs during wine maturation and ageing, leads to wines with more lightness. $C_{\mathrm{ab}}^{*}$ and $s_{\mathrm{uv}}^{*}$ correlate in the same way and are also highly correlated with anthocyanins and with vinyl-anthocyanins. Regarding hue $\left(h_{\mathrm{ab}}\right)$, there is a negative correlation between anthocyanins and $h_{\mathrm{ab}}$ in T and $\mathrm{W}$ wines, which means that an increase of these compounds results in a bluish hue. Pyranoanthocyanins correlate positively with this same parameter meaning that an increase of these compounds provokes an increase in the hue value (towards red-oranges hues). No correlations between total anthocyanin derived pigments, and colour parameters are observed. Anthocyanin vinyl derivatives show the greatest number of correlations which have opposite sign to the Vitisins A and B (when the correlation exists) in the analysis. This behaviour indicates that these two types of pyranoanthocyanins contribute to wine colour in a different way.

\subsection{Principal component analysis (PCA)}

PCA can be used as a form of variable reduction, reducing the large dataset to a much smaller more manageable one which can be interpreted more easily. Also, latent structures between the investigated variables can be observed [32].

In this study, PCA was used in order to identify a small number of factors that explain most of the variance observed in the variables and that could differentiate between wines.

Fig. 3 shows the projection of the 132 wine samples in a two-dimensional space defined by the first and third principal components. From the visual inspection of the PCA score plot it was not possible to adequately distinguish between the different types of wines (Plot $\mathrm{A}$ ). If values corresponding to $\mathrm{M}$ and $\mathrm{W}$ are removed from the plot (Plot A1), it can be seen that PC3 allows the distinction between $\mathrm{T}$ (on the bottom, negative or near zero scores) and G (positive scores), whereas $\mathrm{M}$ and $\mathrm{W}$ wines are located between them (Plot A2). These principal components explain $56 \%$ of variation in the data set $(\mathrm{PC} 1=47 \%$ and $\mathrm{PC} 3=9 \%$ ).

An attempt was made to determine whether the latent variables could also reveal other sources of differentiation. Considering the different winemaking steps in the PCA score plot it is noticeable that differentiation between steps is influenced by PC1 (Fig. 4). Therefore, one can conclude that pigments appeared to be much more useful in describing the steps of the winemaking than in describing the type ( $\mathrm{M}$ or $\mathrm{W}$ ) of wine.

The second principal component (PC2) describes $14 \%$ of the variability in the data but no known tendency was observed in this component, probably because it was related with environmental conditions or other factors about which we do not have information. That is why it was not represented.

The original variables most related to the first and third principal components were for PC1: "Total anthocyanidins 3-O-glucoside content", "Total anthocyanin content", "Total pigment content" and "malvidin $3 \mathrm{O}$-glucoside content". For PC3: "Pigments derived from condensation between anthocyanins and flavanols" (direct-linked and ethyl-linked compounds), "Delphinidin 3,5-diglucoside", "Delphinidin 3,7-diglucoside" and the colour parameter " $b$ ". 


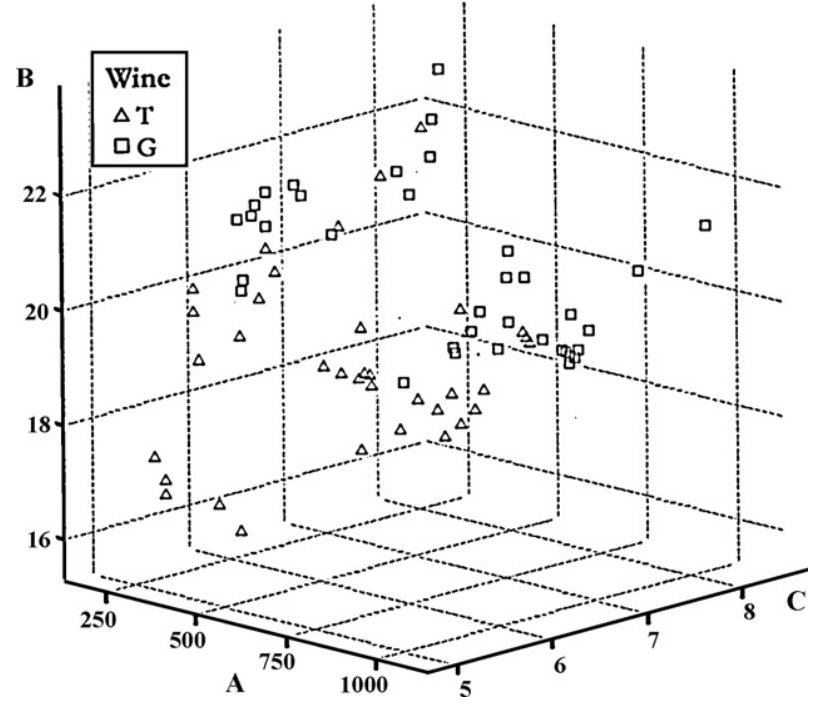

Fig. 6. Projection of the wine samples on the 3D space considering one of the first original variables most related to PC1 (A: anthocyanins 3-glucosides) and two of the first original variables most related to PC3 (B: total condensation products; C: delphinidin 3,5-diglucoside).

Fig. 5 shows the plot of the wine samples considering only the first original variables most related to PC1 and PC3 (anthocyanidins-3-O-glucosides and pigments derived from condensation between anthocyanins and flavanols). This representation does not allow distinctions between $\mathrm{T}$ and $\mathrm{G}$ wines (plot A) but it does show the differences between the winemaking steps (plot B). Nevertheless, considering one more original variable most related to PC3 (delphinidin-3,5-diglucoside) and plotting 3D, differences between $G$ and $T$ can be observed (Fig. 6).

\section{Conclusions}

Pigment extraction and retention in Tempranillo wines seems to be increased by the incorporation of the Graciano variety during the prefermentative maceration step and may be linked to the fact that the flavanols from grape skins of the Graciano variety are better copigments than those of Tempranillo. The wine thus obtained had colour parameters closer to the $G$ wine than to the $T$ wine. $T, G$ and $M$ had different colours detectable by the human eye $\left(\Delta E_{\mathrm{ab}}^{*} \geq 3\right)$ while $\mathrm{T}$ and $\mathrm{W}$ had indistinguishable colours even when using analytical methods $\left(\Delta E_{\mathrm{ab}}^{*} \leq 1\right)$. Data processing through multivariate analysis (PCA) has been demonstrated as a useful technique to separate $\mathrm{T}$ and $G$ wine samples according to the variety and, considering the whole wine samples investigated, to differentiate between different winemaking steps. PC3, whose original most related variable was the content in pigments derived from condensation between anthocyanins and flavanols, allows the distinction between $\mathrm{T}$ and $\mathrm{G}$ wines, whereas PC1, whose original most related variable was the content in anthocyanidins-3-O-glucosides, allows the distinction between the different steps of the winemaking process.

\section{Acknowledgements}

Thanks are due to the Spanish MICINN and FEDER for financial support (projects ref. AGL2005-07245-C03-01 and AGL200805569-C02-01) and to Bodegas RODA S.A. (Haro, La Rioja, Spain) for supplying the wine samples. We would also like to thank Mr. G.H. Jenkins for his help with the English version of the manuscript.

\section{References}

[1] L. Jurd, Am. J. Enol. Vitic. 20 (1969) 191.

[2] T.C. Somers, Phytochemistry 10 (1971) 2175.

[3] S. Asen, R.N. Stewart, K.H. Norris, Phytochemistry 11 (1972) 1139.

[4] R. Boulton, Am. J. Enol. Vitic. 52 (2001) 67.

[5] R. Brouillard, O. Dangles, Food Chem. 51 (1994) 365.

[6] D.G. Bloonfield, D.A. Heatherbell, M.S. Pour Nikfardjam, Mitt. Klosterneuburg 53 (2003) 195.

[7] J. Darias-Martín, M. Carrillo, E. Díaz, R.B. Boulton, Food Chem. 73 (2001) 217.

[8] J. Darias-Martín, B. Martín-Luis, M. Carrillo-López, R. Lamuela-Raventós, C. Díaz-Romero, R.B. Boulton, J. Agric. Food Chem. 50 (2002) 2062.

[9] M.V. Moreno-Arribas, M.C. Polo, Wine Chemistry and Biochemistry, Springer, 2008.

[10] C. Lorenzo, F. Pardo, A. Zalacain, G.L. Alonso, M.R. Salinas, J. Agric. Food Chem. 53 (2005) 7609.

[11] M. Monagas, B. Bartolomé, C. Gómez-Cordovés, LWT-Food Sci. Technol. 39 (2006) 1133.

[12] S. González-Manzano, M. Dueñas, J.C. Rivas-Gonzalo, M.T. Escribano-Bailón, C Santos-Buelga, Food Chem. 114 (2009) 649.

[13] D.L. Massart, B.G.M. Vandeginste, L.M.C. Buydens, S. De Jong, P.J. Lewi, J. Smeyers-Verbeke, Handbook of Chemometrics and Qualimetrics. Part A, Elsevier, Amsterdam, The Netherlands, 1997.

[14] B.G.M. Vandeginste, D.L. Massart, L.M.C. Buydens, S. De Jong, P.J. Lewi, J. Smeyers-Verbeke, Handbook of Chemometrics and Qualimetrics. Part B, Elsevier, Amsterdam, The Netherlands, 1998.

[15] S. Kallithraka, I.S. Arvanitoyannis, P. Kefalas, A. El-Zajouli, E. Soufleros, E. Psarra, Food Chem. 17 (2001) 501.

[16] C.J. Bevin, R.G. Dambergs, A.J. Fergusson, D. Cozzolino, Anal. Chim. Acta 621 (2008) 19.

[17] J.S. Câmara, M. Arminda Alves, J.C. Marques, Talanta 68 (2006) 1512.

[18] F.J. Heredia, C. Álvarez, M.L. Gonzalez-Miret, A. Ramirez, CromaLab ${ }^{\circledR}$, análisis de color, Registro General de la Propiedad Intelectual SE-1052-04, Sevilla, Spain, 2004.

[19] M. Melgosa, E. Hita, A.J. Poza, D.H. Alman, R.S. Berns, Color Res. Appl. 22 (1997) 148

[20] J.A. Martínez, M. Melgosa, M.M. Pérez, E. Hita, A.I. Negueruela, Food Sci. Technol. Int. 7 (2001) 439.

[21] C. Alcalde-Eon, M.T. Escribano-Bailón, C. Santos-Buelga, J.C. Rivas-Gonzalo Anal. Chim. Acta 563 (2006) 238

[22] H. Wang, E.J. Race, A.J. Shrikhande, J. Agric. Food Chem. 51 (2003) 7989

[23] C. Alcalde-Eon, E. Boido, F. Carrau, E. Dellacassa, J.C. Rivas-Gonzalo, Am. J. Enol. Vitic. 57 (2006) 449.

[24] P. Valentão, R.M. Seabra, G. Lopes, L.R. Silva, V. Martins, M.E. Trujillo, E Velázquez, P.B. Andrade, Food Chem. 100 (2007) 64.

[25] P. Dugo, L. Mondello, G. Errante, G. Zappia, G. Dugo, J. Agric. Food Chem. 49 (2001) 3987.

[26] Ø.M. Andersen, J. Food Sci. 52 (1987) 665

[27] J. Oszmianski, J.C. Sapis, J. Food Sci. 53 (1988) 1241

[28] M.T. Escribano-Bailón, C. Santos-Buelga, G.L. Alonso, M.R. Salinas, Phytochem Anal. 13 (2002) 354.

[29] A. Baldi, A. Romani, N. Mulinacci, F.F. Vincieri, B. Casetta, J. Agric. Food Chem. 43 (1995) 2104.

[30] A. Heier, W. Blaas, A. Droß, R. Wittkowski, Am. J. Enol. Vitic. 53 (2002) 78.

[31] S. Vidal, E. Meudec, V. Cheynier, G. Skouroumounis, Y. Hayasaka, J. Agric. Food Chem. 52 (2004) 7144.

[32] R.G. Brereton, Chemometrics: Data Analysis for the Laboratory and Chemical Plant, John Wiley \& Sons Ltd., Chichester, England, 2003. 